\title{
ERROR ESTIMATES WITH SMOOTH AND NONSMOOTH DATA FOR A FINITE ELEMENT METHOD FOR THE CAHN-HILLIARD EQUATION
}

\author{
CHARLES M. ELLIOTT AND STIG LARSSON
}

\begin{abstract}
A finite element method for the Cahn-Hilliard equation (a semilinear parabolic equation of fourth order) is analyzed, both in a spatially semidiscrete case and in a completely discrete case based on the backward Euler method. Error bounds of optimal order over a finite time interval are obtained for solutions with smooth and nonsmooth initial data. A detailed study of the regularity of the exact solution is included. The analysis is based on local Lipschitz conditions for the nonlinearity with respect to Sobolev norms, and the existence of a Ljapunov functional for the exact and the discretized equations is essential. A result concerning the convergence of the attractor of the corresponding approximate nonlinear semigroup (upper semicontinuity with respect to the discretization parameters) is obtained as a simple application of the nonsmooth data error estimate.
\end{abstract}

\section{INTRODUCTION}

The Cahn-Hilliard equation

$$
u_{t}+\Delta^{2} u-\Delta \phi(u)=0, \quad x \in \Omega \subset \mathbf{R}^{3}, t>0,
$$

where typically $\phi(u)=u^{3}-u$, together with appropriate boundary and initial conditions, is a phenomenological model for phase separation and spinodal decomposition. The boundary conditions are such that the fourth-order differential operator in (1.1) can be written as the square of a second-order elliptic operator. Relying on this fact, we study numerical schemes for (1.1), which for the approximation of the spatial variables are based on standard Galerkin finite element methods for second-order elliptic problems. We discuss spatially semidiscrete schemes as well as a completely discrete scheme based on the backward Euler method.

A semidiscrete finite element method (with numerical quadrature) of this type for the Cahn-Hilliard equation was first introduced and analyzed by Elliott, French, and Milner [7]. Completely discrete schemes based on the same idea

Received March 19, 1991.

1991 Mathematics Subject Classification. Primary 65M15, 65M60.

Key words and phrases. Cahn-Hilliard equation, nonlinear, semigroup, smoothing property, finite element, backward Euler method, error estimate, nonsmooth data, upper semicontinuity, attractor.

This work was partially supported by the SERC. The second author was also supported by the Swedish National Board for Technical Development (STUF). 
were discussed by $\mathrm{Du}$ and Nicolaides [5] and Du [4]. For numerical schemes based on other approximations of the fourth-order elliptic operator we refer to Elliott and Zheng [8] (conforming elements in 1-D) and Elliott and French [6] (nonconforming elements in 2-D).

In these works the analysis is restricted to solutions which are bounded uniformly in time, so that one may essentially assume that the nonlinearity $\phi$ satisfies a global Lipschitz condition. Because of the lack of a maximum principle this means that one has to prove (or assume) that the solution is sufficiently smooth depending on the number of space dimensions.

The purpose of the present work is to prove error bounds that are optimal both in the order of convergence and in the regularity assumed of the initial data. In particular, we would like to allow initial data of low regularity (compared to the number of derivatives occurring in equation (1.1)). The reason for this is the existence of a Ljapunov functional for equation (1.1) and its discrete counterparts, which yields an a priori bound, uniform in time, for the $H^{1}$ norm of the solution and for the discrete approximations considered. The Sobolev space $H^{1}(\Omega)$ is therefore a natural space in which to prescribe initial data.

Moreover, error bounds for solutions with nonsmooth initial data have interesting applications in the study of the longtime behavior of discrete solutions, see Heywood and Rannacher [12], Hale, Lin, and Raugel [10] and Kloeden and Lorenz [14]. As an example of this, we prove a result concerning the convergence of the attractor of the corresponding approximate nonlinear semigroup. More precisely, we demonstrate that the discrete attractor is upper semicontinuous with respect to the discretization parameters.

With initial data in $H^{1}(\Omega)$, the solution is not bounded uniformly in time (except in the case of one space dimension). Instead, we base our analysis on uniform bounds in the $H^{1}$ norm for the exact and discrete solutions and local Lipschitz conditions for the nonlinearity $\phi$. These are typically of the form

$$
\|\phi(u)-\phi(v)\|_{X} \leq C\left(\|u\|_{H^{1}},\|v\|_{H^{1}}\right)\|u-v\|_{Y},
$$

where $\|\cdot\|_{X},\|\cdot\|_{Y}$ are appropriate Sobolev norms.

Nonsmooth data error estimates for finite element methods have been proved earlier by Johnson, Larsson, Thomée, and Wahlbin [13], Crouzeix, Thomée, and Wahlbin [3] and Crouzeix and Thomée [2] in the context of a semilinear parabolic problem of second order with globally Lipschitz continuous nonlinearity. Similar results were obtained by Helfrich [11] in an abstract framework, using local Lipschitz conditions. See also Heywood and Rannacher [12] for related results in the context of the Navier-Stokes equations.

Loosely speaking, our main result (Theorem 6.5) states the following: Let $u_{h}$ be the spatially semidiscrete approximation using a finite element method of order $r$ and with mesh parameter $h$, and let the initial approximation be chosen as the $L_{2}$ projection of the exact initial value $u_{0}$. Then for $r=2$ or 3 (piecewise linear or quadratic finite elements) we have

$$
\left\|u_{h}(t)-u(t)\right\|_{L_{2}} \leq C\left(u_{0}, T\right) h^{r} t^{-\frac{r-\alpha}{4}}, \quad 0<t \leq T,
$$

for $1 \leq \alpha \leq r$, provided that $u_{0}$ has $\alpha$ derivatives in $L_{2}$ (together with appropriate boundary conditions). An analogous result is obtained in the completely discrete case (Theorem 7.2). The restrictions $r=2$ or 3 and $\alpha \geq 1$ are probably due to our method of proof, but in the light of a counterexample in 
$[13,3]$ some restriction of this type might be expected. We have, however, not been able to adapt this counterexample to the present situation. See also Remark 2 of $\S 5$ below.

The outline of the paper is as follows. In $\S 2$ we present three initial-boundary value problems for the Cahn-Hilliard equation and put them into a common abstract framework. In $\S 3$ we introduce spatially semidiscrete and completely discrete finite element methods for these problems. In $\S 4$ we state a result concerning the regularity of the exact solution, which is needed in the subsequent error analysis. Its proof is given in an Appendix in the Supplement section of this issue. In $\S 5$ we estimate the difference between the exact solution and the solution of a discrete linear auxiliary problem. This analysis is based on energy estimates. In $\S 6$ we prove error estimates for the spatially semidiscrete approximation, and in $\S 7$ we do the same for the fully discrete approximation. This analysis is based on semigroup techniques. Finally, in $\S 8$ we demonstrate the existence of global attractors for the nonlinear semigroups defined by the Cahn-Hilliard equation and its approximations, and prove a result concerning the convergence of the discrete attractors.

\section{The CAHN-Hilliard equation}

Let $\Omega$ be a bounded domain in $\mathbf{R}^{d}$ for $d \leq 3$ with a sufficiently smooth boundary. We consider the finite element approximation of the following initialboundary value problems: Find $u(x, t)$ for $x \in \Omega, t>0$, such that

$$
\begin{array}{ll}
u_{t}-\Delta(-\Delta u+\phi(u))=0, & x \in \Omega, t>0, \\
u(x, 0)=u_{0}(x), & x \in \Omega,
\end{array}
$$

subject to one of the three sets of boundary conditions,

$$
\begin{array}{lll}
(2.3-\mathrm{a}) & u=0,-\Delta u+\phi(u)=0, & x \in \partial \Omega, t>0, \\
(2.3-\mathrm{b}) & \frac{\partial u}{\partial \nu}=0, \frac{\partial}{\partial \nu}(-\Delta u+\phi(u))=0, & x \in \partial \Omega, t>0, \\
(2.3-\mathrm{c}) & u\left(x+L e_{i}, t\right)=u(x, t), & x \in \partial \Omega, t>0, i=1, \ldots, d .
\end{array}
$$

Here, $\phi$ is a given polynomial satisfying the structural assumptions

$$
\begin{aligned}
& \phi(s)=\psi^{\prime}(s), \quad \text { degree } \psi=2 p, \\
& \psi(s) \geq c_{0}|s|^{2 p}-c_{1}, \quad \psi^{\prime \prime}(s) \geq-\beta^{2} \quad \forall s \in \mathbf{R},
\end{aligned}
$$

where $c_{0}>0$ and $2 \leq p<\infty$ if $d \leq 2, p=2$ if $d=3$. In the case of the Dirichlet boundary conditions (2.3-a) we make the additional assumption that $\phi(0)=0$.

In (2.3-b) we have used the notation $\partial / \partial \nu$ for the outward normal derivative, and in (2.3-c), the case of periodic boundary conditions, we understand $\Omega$ to be a "cube" $(0, L)^{d}$ with $e_{i}$ denoting the unit vector in the direction of the $x_{i}$-axis.

The differential equation in (2.1) is known as the Cahn-Hilliard equation. It arises in continuum models of phase separation and spinodal decomposition, cf. Cahn and Hilliard [1]. The field variable $u$ is a scaled concentration of one species in a binary mixture and the "free energy" $\psi$ is a double well potential. A typical example for $\psi$ is $\psi(s)=\frac{1}{4}\left(s^{2}-\beta^{2}\right)^{2}$ with $\phi(s)=s\left(s^{2}-\beta^{2}\right)$. 
In order to put these three initial-boundary value problems in a common abstract framework, we introduce some notation. Let $\|\cdot\|$ and $(\cdot, \cdot)$ denote the usual norm and inner product in $L_{2}=L_{2}(\Omega)$, and let $H^{s}=H^{s}(\Omega)$ with norms $\|\cdot\|_{s}$ be the usual Sobolev spaces.

For the no flux and the periodic boundary conditions $(2.3-b, c)$, it is easy to see that a sufficiently smooth solution of (2.1), (2.2) satisfies conservation of mass,

$$
\int_{\Omega} u(x, t) d x=\int_{\Omega} u_{0}(x) d x, \quad t \geq 0 .
$$

Introducing the change of variables $\tilde{u}=u-\overline{u_{0}}$ and $\tilde{\psi}(\tilde{u})=\psi\left(\tilde{u}+\overline{u_{0}}\right)$, where $\overline{u_{0}}$ denotes the average of $u_{0}$, we see that the equations $(2.1),(2.2),(2.3-\mathrm{b}, \mathrm{c})$ and the structural assumptions (2.4) remain unchanged. Henceforth, for the boundary conditions $(2.3-\mathrm{b}, \mathrm{c})$, we assume that the initial datum satisfies $\int_{\Omega} u_{0}(x) d x=0$. For these boundary conditions we let $H$ denote the subspace of $L_{2}$ which is orthogonal to the constants, $H=\left\{v \in L_{2}:(v, 1)=0\right\}$, and let $P$ be the orthogonal projection of $L_{2}$ onto $H$. Clearly then $P f=f-\bar{f}$. For the Dirichlet boundary conditions (2.3-a), we let $H=L_{2}$ and $P=I$. We then define the linear operator $A=-\Delta$ with domain of definition

$$
\begin{aligned}
& \mathscr{D}(A)=\left\{v \in H^{2}: v=0 \text { on } \partial \Omega\right\}, \\
& \mathscr{D}(A)=\left\{v \in H^{2} \cap H: \frac{\partial v}{\partial \nu}=0 \text { on } \partial \Omega\right\}, \\
& \mathscr{D}(A)=\left\{v \in H^{2} \cap H: v\left(x+L e_{i}\right)=v(x) \text { for } x \in \partial \Omega, i=1, \ldots, d\right\},
\end{aligned}
$$

for the three sets of boundary conditions, respectively. Then $A$ is a selfadjoint positive definite densely defined operator on $H$, and (2.1)-(2.3) may be written as an abstract initial value problem

$$
\begin{aligned}
& u_{t}+A^{2} u+A P \phi(u)=0, \quad t>0, \\
& u(0)=u_{0} .
\end{aligned}
$$

By spectral theory we may also define the spaces $\dot{H}^{s}=\mathscr{D}\left(A^{\frac{s}{2}}\right)$ with norms $|v|_{s}=\left\|A^{\frac{s}{2}} v\right\|$ for real $s$. It is well known that, for integer $s \geq 0, \dot{H}^{s}$ is a subspace of $H^{s} \cap H$ characterized by certain boundary conditions, and that the norms $|\cdot|_{s}$ and $\|\cdot\|_{s}$ are equivalent on $\dot{H}^{s}$. This can be proved by means of the spectral theorem and trace inequalities, see Thomée [18, p. 34] for a proof in the case of the Dirichlet boundary condition. In particular, we have

$$
\begin{aligned}
& \dot{H}^{1}=\left\{v \in H^{1}: v=0 \text { on } \partial \Omega\right\}, \\
& \dot{H}^{1}=H^{1} \cap H, \\
& \dot{H}^{1}=\left\{v \in H^{1} \cap H: v\left(x+L e_{i}\right)=v(x) \text { for } x \in \partial \Omega, i=1, \ldots, d\right\},
\end{aligned}
$$

for the three sets of boundary conditions, respectively, and the norm $|v|_{1}=$ $\left\|A^{\frac{1}{2}} v\right\|=\|\nabla v\|$ is equivalent to $\|v\|_{1}$ on $\dot{H}^{1}$. Apart from this, we shall only need the inequality

$$
\|v\|_{s} \leq C_{s}|v|_{s}, \quad v \in \dot{H}^{s}, s \geq 0,
$$

which follows by interpolation between the corresponding inequalities with integer $s$. 
We also define $G: H \rightarrow \dot{H}^{2}$ to be the inverse of $A$. It is convenient to extend it to all of $L_{2}$ by $G f=G P f$ for $f \in L_{2}$. Thus, $v=G f$ if and only if $A v=P f$, or equivalently

$$
v \in \dot{H}^{1}, \quad(\nabla v, \nabla \chi)=(f, \chi) \quad \forall \chi \in \dot{H}^{1} .
$$

Clearly, $G$ is selfadjoint positive semidefinite on $L_{2}$ and positive definite on $H$.

We next derive an a priori bound in the $H^{1}$ norm for solutions of (2.5). This bound (and its discrete counterparts) will be basic to all of our analysis below. Applying $G$ to $(2.5)$, we have

$$
G u_{t}+A u+P \phi(u)=0,
$$

and taking the inner product of this with $u_{t}$, we obtain

$$
\left(G u_{t}, u_{t}\right)+\frac{1}{2} D_{t}|u|_{1}^{2}+D_{t} \int_{\Omega} \psi(u) d x=0 .
$$

Setting $V(u)=\frac{1}{2}|u|_{1}^{2}+\int_{\Omega} \psi(u) d x$ ("the free energy functional"), we conclude

$$
\int_{0}^{t}\left\|G^{\frac{1}{2}} u_{t}\right\|^{2} d s+V(u(t))=V\left(u_{0}\right), \quad 0 \leq t<\infty,
$$

provided that $u_{0} \in \dot{H}^{1}$. In view of the structural assumptions (2.4) it follows that $V$ is a Ljapunov functional for the initial value problem (2.5) (see $\S 8$ below for the definition of this concept). Moreover, by the Sobolev imbedding of $H^{1}$ into $L_{2 p}$ (where $p$ is as in (2.4)) the identity (2.7) implies an a priori bound: If $u_{0} \in \dot{H}^{1}$ with $\left\|u_{0}\right\|_{1} \leq R$, then

$$
\|u(t)\|_{1} \leq C(R), \quad 0 \leq t<\infty .
$$

In the sequel we shall always assume that $u_{0} \in \dot{H}^{1}$ (at least), so that (2.8) holds. We also note that the derivative of $V$ ("the chemical potential") is given by $w=V^{\prime}(u)=A u+P \phi(u)=-G u_{t}$.

Finally, we let $E(t)=\exp \left(-t A^{2}\right)$ denote the analytic semigroup generated by $-A^{2}$. Much of our analysis will be based on the variations of constants formula,

$$
u(t)=E(t) u_{0}-\int_{0}^{t} E(t-s) A P \phi(u(s)) d s,
$$

for solutions of (2.5).

\section{THE FINITE ELEMENT METHOD}

For the approximation of the Cahn-Hilliard equation we assume that we have a family $\left\{S_{h}\right\}_{h>0}$ of finite-dimensional approximating subspaces of $H^{1}$. At the end of this section we formulate the approximation assumption upon which we shall base our error analysis. But first we formulate our discrete equations.

Consider, to begin with, the no flux boundary conditions (2.3-b). Recalling the usual weak formulation of the corresponding initial-boundary value problem, we state the following semidiscrete problem: Find $u_{h}(t), v_{h}(t) \in S_{h}$ such that

$$
\begin{array}{ll}
\left(u_{h, t}, \chi\right)+\left(\nabla v_{h}, \nabla \chi\right)=0 & \forall \chi \in S_{h}, t>0, \\
\left(v_{h}, \chi\right)=\left(\nabla u_{h}, \nabla \chi\right)+\left(\phi\left(u_{h}\right), \chi\right) & \forall \chi \in S_{h}, t>0, \\
u_{h}(0)=u_{0 h}, &
\end{array}
$$


where $u_{0 h} \in S_{h}$ is a suitable approximation of $u_{0} \in \dot{H}^{1}$. Since we are assuming that $\overline{u_{0}}=0$, it is natural to assume that $\overline{u_{0 h}}=0$, too. It is easy to see that this can be achieved, e.g., by taking $u_{0 h}$ to be the orthogonal projection of $u_{0} \in \dot{H}^{1}$ onto $S_{h}$ with respect to the $L_{2}$ inner product, or with respect to the $H^{1}$ inner product. Let now

$$
\dot{S}_{h}=\left\{\chi \in S_{h}:(\chi, 1)=0\right\} .
$$

It is immediate from (3.1) that $u_{h}(t) \in \dot{S}_{h}$ if $u_{0 h} \in \dot{S}_{h}$. Therefore, $u_{h}$ can equivalently be obtained from the following equations: Find $u_{h}(t), w_{h}(t) \in \dot{S}_{h}$ such that

$$
\begin{array}{ll}
\left(u_{h}, \chi\right)+\left(\nabla w_{h}, \nabla \chi\right)=0 & \forall \chi \in \dot{S}_{h}, t>0, \\
\left(w_{h}, \chi\right)=\left(\nabla u_{h}, \nabla \chi\right)+\left(\phi\left(u_{h}\right), \chi\right) & \forall \chi \in \dot{S}_{h}, t>0, \\
u_{h}(0)=u_{0 h}, &
\end{array}
$$

where now $u_{0 h} \in \dot{S}_{h}$ is an approximation of $u_{0} \in \dot{H}^{1}$. (The relation between $w_{h}$ and $v_{h}$ is $w_{h}=v_{h}-\overline{v_{h}}$.) Equivalently, we may write this as

$$
\begin{aligned}
& u_{h, t}+A_{h}^{2} u_{h}+A_{h} P_{h} \phi\left(u_{h}\right)=0, \quad t>0, \\
& u_{h}(0)=u_{0 h},
\end{aligned}
$$

where the operator $A_{h}: \dot{S}_{h} \rightarrow \dot{S}_{h}$ (the "discrete Laplacian") is defined by

$$
\left(A_{h} \chi, \eta\right)=(\nabla \chi, \nabla \eta) \quad \forall \chi, \eta \in \dot{S}_{h},
$$

and $P_{h}: L_{2} \rightarrow \dot{S}_{h}$ is the orthogonal projection. Clearly, $A_{h}$ is selfadjoint positive definite, and we let $G_{h}$ denote its inverse. As for $G$, it is convenient to extend $G_{h}$ to all of $L_{2}$ by $G_{h} f=G_{h} P_{h} f$ for $f \in L_{2}$. Thus, $v_{h}=G_{h} f$ if and only if $A_{h} v_{h}=P_{h} f$, or equivalently

$$
v_{h} \in \dot{S}_{h}, \quad\left(\nabla v_{h}, \nabla \chi\right)=(f, \chi) \quad \forall \chi \in \dot{S}_{h} .
$$

We note that, thus defined, $G_{h}$ is selfadjoint positive semidefinite on $L_{2}$ and positive definite on $\dot{S}_{h}$. We also record the facts that $\left\|A_{h}^{\frac{1}{2}} \chi\right\|=\|\nabla \chi\|=|\chi|_{1}$ for all $\chi \in \dot{S}_{h}$, and that for the "discrete chemical potential" $w_{h}$ in (3.2), we have $w_{h}=A_{h} u_{h}+P_{h} \phi\left(u_{h}\right)=-G_{h} u_{h, t}$.

The above refers to the no flux boundary conditions. In the case of the Dirichlet boundary conditions (2.3-a), we define instead

$$
\dot{S}_{h}=\left\{\chi \in S_{h}: \chi=0 \text { on } \partial \Omega\right\},
$$

and for the periodic boundary conditions (2.3-c), we set

$$
\dot{S}_{h}=\left\{\chi \in S_{h}: \chi\left(x+L e_{i}\right)=\chi(x) \text { on } \partial \Omega, i=1, \ldots, d\right\} .
$$

Starting with (3.2), we then reiterate the above arguments and definitions. The initial value problem (3.3) is thus a common framework for our semidiscrete approximations of the three initial-boundary value problems $(2.1)-(2.3)$.

We now derive a discrete counterpart to the a priori bound (2.8). In fact, $V(u)=\frac{1}{2}|u|_{1}^{2}+\int_{\Omega} \psi(u) d x$ is a Ljapunov functional for (3.3), too. To see this, we argue in the same way as in the proof of (2.7) and obtain

$$
\int_{0}^{t}\left\|G_{h}^{\frac{1}{2}} u_{h, t}\right\|^{2} d s+V\left(u_{h}(t)\right)=V\left(u_{0 h}\right), \quad 0 \leq t<\infty
$$


which leads to the a priori bound: If $u_{0 h} \in \dot{S}_{h}$ with $\left\|u_{0 h}\right\|_{1} \leq R$, then

$$
\left\|u_{h}(t)\right\|_{1} \leq C(R), \quad 0 \leq t<\infty .
$$

With $E_{h}(t)=\exp \left(-t A_{h}^{2}\right)$ we have the variations of constants formula,

$$
u_{h}(t)=E_{h}(t) u_{0 h}-\int_{0}^{t} E_{h}(t-s) A_{h} P_{h} \phi\left(u_{h}(s)\right) d s,
$$

for solutions of (3.3).

We next formulate a fully discrete approximation based on the backward Euler method. This means that we replace the time-derivative in (3.2) or (3.3) by a backward difference quotient $\bar{\partial}_{t} U_{n}=\left(U_{n}-U_{n-1}\right) / k$, where $k$ is the time step and $U_{n}$ is the approximation to $u$ at time $t_{n}=n k, n=0,1,2, \ldots$. We thus seek $U_{n} \in \dot{S}_{h}$ such that

$$
\begin{aligned}
& \bar{\partial}_{t} U_{n}+A_{h}^{2} U_{n}+A_{h} P_{h} \phi\left(U_{n}\right)=0, \quad t_{n}>0, \\
& U_{0}=u_{0 h} .
\end{aligned}
$$

Again, it turns out that the functional $V$ is a Ljapunov functional for (3.6). In fact, arguing as in the proof of (2.7), we obtain

$$
\left(G_{h} \bar{\partial}_{t} U_{n}, \bar{\partial}_{t} U_{n}\right)+\left(A_{h} U_{n}, \bar{\partial}_{t} U_{n}\right)+\left(\phi\left(U_{n}\right), \bar{\partial}_{t} U_{n}\right)=0 .
$$

Here,

$$
\left(A_{h} U_{n}, \bar{\partial}_{t} U_{n}\right)=\frac{1}{2} \bar{\partial}_{t}\left|U_{n}\right|_{1}^{2}+\frac{1}{2} k\left|\bar{\partial}_{t} U_{n}\right|_{1}^{2} .
$$

Recalling the condition $\psi^{\prime \prime}(s) \geq-\beta^{2}$ in (2.4), we obtain that

$$
\psi^{\prime}(r)(r-s) \geq \psi(r)-\psi(s)-\frac{1}{2} \beta^{2}(r-s)^{2},
$$

so that

$$
\left(\phi\left(U_{n}\right), \bar{\partial}_{t} U_{n}\right) \geq \bar{\partial}_{t} \int_{\Omega} \psi\left(U_{n}\right) d x-\frac{1}{2} k \beta^{2}\left\|\bar{\partial}_{t} U_{n}\right\|^{2}
$$

Hence,

$$
\begin{aligned}
& \left\|G_{h}^{\frac{1}{2}} \bar{\partial}_{t} U_{n}\right\|^{2}+\frac{1}{2} k\left|\bar{\partial}_{t} U_{n}\right|_{1}^{2}+\bar{\partial}_{t} V\left(U_{n}\right) \\
& \quad \leq \frac{1}{2} k \beta^{2}\left\|\bar{\partial}_{t} U_{n}\right\|^{2} \leq \frac{1}{8} k \beta^{4}\left\|G_{h}^{\frac{1}{2}} \bar{\partial}_{t} U_{n}\right\|^{2}+\frac{1}{2} k\left|\bar{\partial}_{t} U_{n}\right|_{1}^{2} .
\end{aligned}
$$

Thus, if $k \leq 4 / \beta^{4}$, this shows that

$$
\frac{1}{2} k \sum_{j=1}^{n}\left\|G_{h}^{\frac{1}{2}} \bar{\partial}_{t} U_{n}\right\|^{2}+V\left(U_{n}\right) \leq V\left(u_{0 h}\right), \quad 0 \leq t_{n}<\infty,
$$

which leads to the a priori bound: If $u_{0 h} \in \dot{S}_{h}$ with $\left\|u_{0 h}\right\|_{1} \leq R$, then

$$
\left\|U_{n}\right\|_{1} \leq C(R), \quad 0 \leq t_{n}<\infty .
$$

This time, the variation of constants formula becomes

$$
U_{n}=E_{k h}^{n} u_{0 h}-k \sum_{j=1}^{n} E_{k h}^{n-j+1} A_{h} P_{h} \phi\left(U_{j}\right),
$$

where $E_{k h}=\left(I+k A_{h}^{2}\right)^{-1}$. 
We conclude this section by formulating an approximation assumption for the spaces $\dot{S}_{h} \subset \dot{H}^{1}$, which will be the basis for our error analysis below. Let $R_{h}: \dot{H}^{1} \rightarrow \dot{S}_{h}$ be the Ritz projection defined by

$$
\left(\nabla\left(R_{h} v-v\right), \nabla \chi\right)=0 \quad \forall \chi \in \dot{S}_{h} .
$$

We assume that, for $r=2$ or $r=3$,

$$
\left|R_{h} v-v\right|_{l} \leq C h^{\beta-l}|v|_{\beta}, \quad-(r-2) \leq l \leq 1, \quad 1 \leq \beta \leq r .
$$

(Recall that $|v|_{-1}=\left\|G^{\frac{1}{2}} v\right\|=\sup _{\chi \in \dot{H}^{1}}|(v, \chi)| /|v|_{1}$.) From this assumption it follows that

$$
\left\|P_{h} v-v\right\| \leq C h^{r}|v|_{r}
$$

The main examples of this situation are obtained by letting $S_{h}$ be the standard piecewise linear $(r=2)$ or piecewise quadratic $(r=3)$ finite element spaces.

\section{EXISTENCE AND REGULARITY OF SOLUTIONS}

We now state a result concerning existence and regularity of solutions to the Cahn-Hilliard equation (2.5). Global existence has been proved by several authors under various assumptions of initial regularity, see, e.g., Nicolaenko, Scheurer, and Temam [15], Temam [17], Elliott and Zheng [8], Zheng [21] and von Wahl [20]. Our error analysis depends on precise regularity estimates for the exact solution, most of which are not available in the literature, and we therefore develop the required results in the following theorem. Our approach is based on the techniques of [20], where global existence of solutions with initial data in $H^{1}$ was shown.

Theorem 4.1. Let $\alpha \in[1,3], \beta \in[0,4), j, l=0,1,2$, with $4 j-2 l+\beta \geq \alpha$, and let $T, R \geq 0$ be arbitrary. If $u_{0} \in \dot{H}^{\alpha}$ with $\left|u_{0}\right|_{\alpha} \leq R$, then equation (2.5) has a unique solution $u$ which belongs to $C\left([0, T], \dot{H}^{\alpha}\right) \cap C^{1}\left((0, T], L_{2}\right)$. Moreover, there is a constant $C=C(T, R, \beta)$ such that

$$
\left\|G^{l} D_{t}^{j} u(t)\right\|_{\beta} \leq C t^{-j+\frac{l}{2}-\frac{\beta-\alpha}{4}}, \quad 0<t \leq T .
$$

The estimate (4.1) means that the solution operator of the nonlinear CahnHilliard equation enjoys (at least to some extent) a smoothing property analogous to that of the analytic semigroup $E(t)$ :

$$
\left|D_{t}^{j} E(t) v\right|_{\beta} \leq C_{\alpha, \beta} t^{-j-\frac{\beta-\alpha}{4}}|v|_{\alpha}, \quad t>0,0 \leq \alpha \leq \beta .
$$

The proof of Theorem 4.1 can be found in the Supplement section of this issue.

\section{ERROR ANALYSIS FOR A LINEAR PROBLEM}

In this section we shall discuss the following linear nonhomogeneous variant of the Cahn-Hilliard equation (2.5): Let $u$ satisfy the initial value problem

$$
\begin{aligned}
u_{t}+A^{2} u & =A P f, \quad t>0, \\
u(0) & =u_{0},
\end{aligned}
$$

together with the regularity assumption that, for some $T>0, \alpha \in[0,3]$, $K>0$,

$$
\left\|G^{l} D_{t}^{j} u(t)\right\|_{\beta} \leq K t^{--j+\frac{l}{2}-\frac{\beta-\alpha}{4}}, \quad 0<t \leq T,
$$

for all $\beta \in[0,3], j, l=0,1,2$ with $4 j-2 l+\beta \geq \alpha$. 
We shall apply this in the following two situations: If $u$ is the solution of (2.5) (i.e., $f=-\phi(u))$, then (5.2) holds with $K=C(T, R)$ whenever $\left|u_{0}\right|_{\alpha} \leq R$ according to Theorem 4.1. If $u(t)=E(t) u_{0}$ (i.e., $\left.f=0\right)$, then we have $K=C\left|u_{0}\right|_{\alpha}$ according to (4.2).

We first consider a semidiscrete finite element approximation $u_{h}(t) \in \dot{S}_{h}$ given by

$$
\begin{aligned}
u_{h, t}+A_{h}^{2} u_{h} & =A_{h} P_{h} f, \quad t>0, \\
u_{h}(0) & =P_{h} u_{0} .
\end{aligned}
$$

We shall estimate the difference between $u_{h}$ and $u$ under the regularity assumption (5.2). This analysis is linear in the sense that $u_{h}$ depends linearly on $u$.

Observe that by applying $G^{2}$ to (5.1) we obtain $G^{2} u_{t}+u=G f$ and, similarly for (5.3), $G_{h}^{2} u_{h, t}+u_{h}=G_{h} f$, where we have used the fact that $G P=G$, $G_{h} P_{h}=G_{h}$. For the difference $e=u_{h}-u$ we then have

$$
\begin{aligned}
G_{h}^{2} e_{t}+e & =\left(G_{h}-G\right) f-\left(G_{h}^{2}-G^{2}\right) u_{t}=\left(G_{h}-G\right)\left(P f-G u_{t}\right)-G_{h}\left(G_{h}-G\right) u_{t} \\
& =\left(G_{h}-G\right) A u-G_{h}\left(G_{h}-G\right) u_{t}=\left(R_{h}-I\right) u-G_{h}\left(R_{h}-I\right) G u_{t},
\end{aligned}
$$

where the identities $P f-G u_{t}=A u, R_{h}=G_{h} A$ have been employed. It follows that

$$
G_{h}^{2} e_{t}+e=\rho+G_{h} \eta, \quad t>0
$$

with

$$
\rho=\left(R_{h}-I\right) u, \quad \eta=-\left(R_{h}-I\right) G u_{t} .
$$

Equation (5.4) is the basis for the estimation of $e$. It is convenient to first give a lemma providing estimates of $\rho$ and $\eta$.

Lemma 5.1. Let $r=2$ or 3 , and let $u$ satisfy (5.1) and (5.2) for some $\alpha \in$ $[0, r]$. Assume that $1 \leq \beta \leq r, 0 \leq \beta-\alpha \leq 2$. Then the following bounds hold for $0<t \leq T$ :

$$
\begin{aligned}
t^{j}\left\|D_{t}^{j} \rho(t)\right\| & \leq C K h^{\beta} t^{-\frac{\beta-\alpha}{4}}, \\
t^{j}\left\|D_{t}^{j} \eta(t)\right\| & \leq C K h^{\beta} t^{-\frac{1}{2}-\frac{\beta-\alpha}{4}}, \\
\|\tilde{\rho}(t)\| & \leq C K h^{\beta} t^{1-\frac{\beta-\alpha}{4}}, \\
\|\tilde{\eta}(t)\| & \leq C K h^{\beta} t^{\frac{1}{2}-\frac{\beta-\alpha}{4}},
\end{aligned}
$$

where $\tilde{\rho}(t)=\int_{0}^{t} \rho(\tau) d \tau, \tilde{\eta}(t)=\int_{0}^{t} \eta(\tau) d \tau$. Moreover,

$$
\begin{aligned}
\int_{0}^{t}\left(\tau\|\rho\|^{2}+\tau^{3}\left\|\rho_{t}\right\|^{2}\right. & \left.+\tau^{-1}\|\tilde{\rho}\|^{2}+\|\tilde{\eta}\|^{2}+\tau^{2}\|\eta\|^{2}+\tau^{4}\left\|\eta_{t}\right\|^{2}\right) d \tau \\
& \leq C K^{2} h^{2 \beta} t^{2-\frac{\beta-\alpha}{2}} .
\end{aligned}
$$

Proof. By (3.10) and (5.2) we have

$$
t^{j}\left\|D_{t}^{j} \rho(t)\right\|=t^{j}\left\|\left(R_{h}-I\right) D_{t}^{j} u(t)\right\| \leq C t^{j} h^{\beta}\left\|D_{t}^{j} u(t)\right\|_{\beta} \leq C K h^{\beta} t^{-\frac{\beta-\alpha}{4}},
$$

which is (5.5). Similarly,

$$
t^{j}\left\|D_{t}^{j} \eta(t)\right\|=t^{j}\left\|\left(R_{h}-I\right) D_{t}^{j} G u_{t}(t)\right\| \leq C t^{j} h^{\beta}\left\|D_{t}^{j} G u_{t}(t)\right\|_{\beta} \leq C K h^{\beta} t^{-\frac{1}{2}-\frac{\beta-\alpha}{4}},
$$


and (5.6) is proved. Using these estimates, we obtain

$$
\|\tilde{\rho}(t)\| \leq \int_{0}^{t}\|\rho(\tau)\| d \tau \leq C K h^{\beta} \int_{0}^{t} \tau^{-\frac{\beta-\alpha}{4}} d \tau=C K h^{\beta} t^{1-\frac{\beta-\alpha}{4}},
$$

and

$$
\|\tilde{\eta}(t)\| \leq \int_{0}^{t}\|\eta(\tau)\| d \tau \leq C K h^{\beta} \int_{0}^{t} \tau^{-\frac{1}{2}-\frac{\beta-\alpha}{4}} d \tau=C K h^{\beta} t^{\frac{1}{2}-\frac{\beta-\alpha}{4}},
$$

provided, in the latter case, that $0 \leq \beta-\alpha<2$. For $\beta-\alpha=2$ we have instead

$$
\begin{aligned}
\|\tilde{\eta}(t)\| & \leq C h^{\beta}\left\|\int_{0}^{t} G u_{t} d \tau\right\|_{\beta} \leq C h^{\beta}\left\|\int_{0}^{t} u_{t} d \tau\right\|_{\beta-2} \\
& =C h^{\beta}\left\|u(t)-u_{0}\right\|_{\alpha} \leq C h^{\beta}\left(\|u(t)\|_{\alpha}+\left\|u_{0}\right\|_{\alpha}\right) \leq C K h^{\beta} .
\end{aligned}
$$

This proves (5.7) and (5.8). Finally, (5.9) is an immediate consequence of the previous bounds.

Lemma 5.2. Let $r=2$ or 3 , let $u$ satisfy (5.1) and (5.2) for some $\alpha \in[0, r]$, and let $u_{h}$ be the solution of (5.3). Assume that $0 \leq r-\alpha \leq 2$. Then

$$
\left\|u_{h}(t)-u(t)\right\|_{l} \leq C K h^{r-l} t^{-\frac{r-\alpha}{4}}, \quad 0<t \leq T, l=0,1 .
$$

Moreover, for the "chemical potential" $w=A u-P f$ and its approximation $w_{h}=A_{h} u_{h}-P_{h} f$, we have

$$
\left\|w_{h}(t)-w(t)\right\| \leq C K h^{r} t^{-\frac{1}{2}-\frac{r-\alpha}{4}}, \quad 0<t \leq T .
$$

We remark that $C$ is independent of $T$.

Proof. Let $\beta$ be as in Lemma 5.1. We first note that by our special choice $P_{h} u_{0}$ of discrete initial value we have $G_{h} e(0)=0$, where $e=u_{h}-u$. In order to prove the case $l=0$ of $(5.10)$, we start out by taking the inner product of (5.4) with $e_{t}$. Using the fact that $G_{h}$ is selfadjoint positive semidefinite on $L_{2}$, we get

$$
\left\|G_{h} e_{t}\right\|^{2}+\frac{1}{2} D_{t}\|e\|^{2}=\left(\rho, e_{t}\right)+\left(\eta, G_{h} e_{t}\right) \leq\left(\rho, e_{t}\right)+\frac{1}{2}\|\eta\|^{2}+\frac{1}{2}\left\|G_{h} e_{t}\right\|^{2},
$$

which shows

$$
\left\|G_{h} e_{t}\right\|^{2}+D_{t}\|e\|^{2} \leq 2\left(\rho, e_{t}\right)+\|\eta\|^{2}
$$

Multiplying this by $t^{2}$,

$$
\begin{aligned}
& t^{2}\left\|G_{h} e_{t}\right\|^{2}+D_{t}\left(t^{2}\|e\|^{2}\right) \\
& \quad \leq 2 t\|e\|^{2}+2 D_{t}\left[t^{2}(\rho, e)\right]-4 t(\rho, e)-2 t^{2}\left(\rho_{t}, e\right)+t^{2}\|\eta\|^{2} \\
& \quad \leq C\left(D_{t}\left[t^{2}(\rho, e)\right]+t\|\rho\|^{2}+t^{3}\left\|\rho_{t}\right\|^{2}+t^{2}\|\eta\|^{2}+t\|e\|^{2}\right),
\end{aligned}
$$

and integrating with respect to $t$, we obtain after a simple kick-back argument

$$
\begin{aligned}
& \int_{0}^{t} \tau^{2}\left\|G_{h} e_{t}\right\|^{2} d \tau+t^{2}\|e\|^{2} \\
& \quad \leq C t^{2}\|\rho\|^{2}+C \int_{0}^{t}\left(\tau\|\rho\|^{2}+\tau^{3}\left\|\rho_{t}\right\|^{2}+\tau^{2}\|\eta\|^{2}+\tau\|e\|^{2}\right) d \tau .
\end{aligned}
$$

Invoking the bounds for $\rho$ and $\eta$ in Lemma 5.1, we conclude that

$$
\int_{0}^{t} \tau^{2}\left\|G_{h} e_{t}\right\|^{2} d \tau+t^{2}\|e\|^{2} \leq C K^{2} h^{2 \beta} t^{2-\frac{\beta-\alpha}{2}}+C \int_{0}^{t} \tau\|e\|^{2} d \tau .
$$


We now have to estimate $\int_{0}^{t} \tau\|e\|^{2} d \tau$, and we therefore multiply (5.4) by $e$ to get

$$
\frac{1}{2} D_{t}\left\|G_{h} e\right\|^{2}+\|e\|^{2}=(\rho, e)+\left(\eta, G_{h} e\right) \leq \frac{1}{2}\|\rho\|^{2}+\frac{1}{2}\|e\|^{2}+\|\eta\|\left\|G_{h} e\right\|,
$$

whence

$$
D_{t}\left\|G_{h} e\right\|^{2}+\|e\|^{2} \leq\|\rho\|^{2}+2\|\eta\|\left\|G_{h} e\right\| .
$$

Multiplication by $t$ now yields

$$
D_{t}\left(t\left\|G_{h} e\right\|^{2}\right)+t\|e\|^{2} \leq t\|\rho\|^{2}+t^{2}\|\eta\|^{2}+2\left\|G_{h} e\right\|^{2},
$$

so that, in view of (5.9),

$$
\begin{aligned}
t\left\|G_{h} e\right\|^{2}+\int_{0}^{t} \tau\|e\|^{2} d \tau & \leq \int_{0}^{t}\left(\tau\|\rho\|^{2}+\tau^{2}\|\eta\|^{2}+2\left\|G_{h} e\right\|^{2}\right) d \tau \\
& \leq C K^{2} h^{2 \beta} t^{2-\frac{\beta-\alpha}{2}}+2 \int_{0}^{t}\left\|G_{h} e\right\|^{2} d \tau .
\end{aligned}
$$

To derive an estimate of $\int_{0}^{t}\left\|G_{h} e\right\|^{2} d \tau$, we integrate (5.4) with respect to $t$, taking $G_{h} e(0)=0$ into account. This yields

$$
G_{h}^{2} e+\tilde{e}=\tilde{\rho}+G_{h} \tilde{\eta}, \quad t>0,
$$

where $\tilde{e}(t)=\int_{0}^{t} e d \tau$, etc. Multiplication by $e=D_{t} \tilde{e}$ gives

$$
\left\|G_{h} e\right\|^{2}+\frac{1}{2} D_{t}\|\tilde{e}\|^{2}=(\tilde{\rho}, e)+\left(\tilde{\eta}, G_{h} e\right) \leq\|\tilde{\rho}\|\|e\|+\frac{1}{2}\|\tilde{\eta}\|^{2}+\frac{1}{2}\left\|G_{h} e\right\|^{2},
$$

which after some simple manipulation leads to

$$
\begin{aligned}
\int_{0}^{t}\left\|G_{h} e\right\|^{2} d \tau+\|\tilde{e}\|^{2} & \leq \int_{0}^{t}\left(\|\tilde{\eta}\|^{2}+2\|\tilde{\rho}\|\|e\|\right) d \tau \\
& \leq \int_{0}^{t}\left(\|\tilde{\eta}\|^{2}+\tau^{-1}\|\tilde{\rho}\|^{2}+\frac{1}{4} \tau\|e\|^{2}\right) d \tau \\
& \leq C K^{2} h^{2 \beta} t^{2-\frac{\beta-\alpha}{2}}+\frac{1}{4} \int_{0}^{t} \tau\|e\|^{2} d \tau,
\end{aligned}
$$

and, upon substitution into the right-hand side of (5.13),

$$
\int_{0}^{t} \tau\|e\|^{2} d \tau \leq C K^{2} h^{2 \beta} t^{2-\frac{\beta-\alpha}{2}}
$$

Taken together, estimates (5.12) and (5.14) yield

$$
\int_{0}^{t} \tau^{2}\left\|G_{h} e_{t}\right\|^{2} d \tau+t^{2}\|e\|^{2} \leq C K^{2} h^{2 \beta} t^{2-\frac{\beta-\alpha}{2}},
$$

and the case $l=0$ of (5.10) follows.

It is now convenient to estimate the difference between $w$ and $w_{h}$. Observe that

$$
w-w_{h}=-G u_{t}+G_{h} u_{h, t}=G_{h}\left(u_{h, t}-u_{t}\right)+\left(G_{h}-G\right) u_{t}=G_{h} e_{t}-\eta,
$$

and hence

$$
\left\|w-w_{h}\right\| \leq\left\|G_{h} e_{t}\right\|+\|\eta\| .
$$


In view of Lemma 5.1 it is therefore sufficient to bound $G_{h} e_{t}$. Differentiating (5.4) with respect to $t$ yields

$$
G_{h}^{2} e_{t t}+e_{t}=\rho_{t}+G_{h} \eta_{t}
$$

Taking the inner product of this equation with $e_{t}$ gives

$$
\frac{1}{2} D_{t}\left\|G_{h} e_{t}\right\|^{2}+\left\|e_{t}\right\|^{2}=\left(\rho_{t}, e_{t}\right)+\left(\eta_{t}, G_{h} e_{t}\right),
$$

and after multiplication by $t^{3}$,

$$
\begin{aligned}
\frac{1}{2} D_{t}\left(t^{3}\left\|G_{h} e_{t}\right\|^{2}\right)+t^{3}\left\|e_{t}\right\|^{2} & =\frac{3}{2} t^{2}\left\|G_{h} e_{t}\right\|^{2}+t^{3}\left(\rho_{t}, e_{t}\right)+t^{3}\left(\eta_{t}, G_{h} e_{t}\right) \\
& \leq C\left(t^{2}\left\|G_{h} e_{t}\right\|^{2}+t^{3}\left\|\rho_{t}\right\|^{2}+t^{4}\left\|\eta_{t}\right\|^{2}\right)+\frac{1}{2} t^{3}\left\|e_{t}\right\|^{2},
\end{aligned}
$$

so that

$$
t^{3}\left\|G_{h} e_{t}\right\|^{2}+\int_{0}^{t} \tau^{3}\left\|e_{t}\right\|^{2} d \tau \leq C \int_{0}^{t}\left(\tau^{2}\left\|G_{h} e_{t}\right\|^{2}+\tau^{3}\left\|\rho_{t}\right\|^{2}+\tau^{4}\left\|\eta_{t}\right\|^{2}\right) d \tau
$$

Combining (5.15) and (5.9) with (5.17), we obtain

$$
t^{3}\left\|G_{h} e_{t}\right\|^{2}+\int_{0}^{t} \tau^{3}\left\|e_{t}\right\|^{2} d \tau \leq C K^{2} h^{2 \beta} t^{2-\frac{\beta-\alpha}{2}} .
$$

Together with (5.16), this implies

$$
\left\|w_{h}(t)-w(t)\right\| \leq C K h^{\beta} t^{-\frac{1}{2}-\frac{\beta-\alpha}{4}},
$$

and the desired bound (5.11) follows.

Finally we estimate the $H^{1}$ norm of $e$ by interpolating between the known bounds for the errors in $u_{h}$ and $w_{h}$. Let $e=\left(u_{h}-R_{h} u\right)+\left(R_{h} u-u\right) \equiv \theta+\rho$. Since, by (3.10) and (5.2),

$$
\|\rho(t)\|_{1} \leq C h^{r-1}\|u(t)\|_{r} \leq C h^{r-1} t^{-\frac{r-\alpha}{4}},
$$

it is sufficient to make the following estimation:

$$
\begin{aligned}
|\theta|_{1}^{2} & =\left(\nabla\left(u_{h}-R_{h} u\right), \nabla\left(u_{h}-R_{h} u\right)\right)=\left(\nabla\left(u_{h}-u\right), \nabla\left(u_{h}-R_{h} u\right)\right) \\
& =\left(A_{h} u_{h}-A u, u_{h}-R_{h} u\right)=\left(w_{h}-w, u_{h}-R_{h} u\right)+\left(P_{h} f-P f, u_{h}-R_{h} u\right) \\
& =\left(w_{h}-w, u_{h}-R_{h} u\right) \leq\left\|w_{h}-w\right\|\left\|u_{h}-R_{h} u\right\| \\
& \leq\left\|w_{h}-w\right\|\left(\left\|u_{h}-u\right\|+\left\|R_{h} u-u\right\|\right) .
\end{aligned}
$$

Hence, in view of (5.18), (5.15) and (5.5), we have

$$
\|\theta(t)\|_{1} \leq C h^{\beta} t^{-\frac{1}{4}-\frac{\beta-\alpha}{4}}, \quad 0<t \leq T,
$$

for $\alpha \leq \beta \leq r$. If $\alpha \leq r-1$, then we can take $\beta=r-1$, and the case $l=1$ of (5.10) follows. If $r-1<\alpha \leq r$, we argue differently. A glance at (5.4) reveals that

$$
G_{h}^{2} \theta_{t}+\theta=-G_{h}^{2} \rho_{t}+G_{h} \eta
$$

or

$$
\theta_{t}+A_{h}^{2} \theta=-P_{h} \rho_{t}+A_{h} P_{h} \eta
$$

An estimation of $\|\theta\|_{1}$ can be based on this equation via the variation of constants formula (cf. the proof of Lemma 6.8 below). We omit the details. 
Remark 1. If we choose $\beta=r$ in (5.19) we obtain a bound of superconvergent order for the gradient of $\theta=u_{h}-R_{h} u$ :

$$
\left\|u_{h}(t)-R_{h} u(t)\right\|_{1} \leq C h^{r} t^{-\frac{1}{4}-\frac{r-\alpha}{4}}, \quad 0<t \leq T .
$$

In the case $d \leq 2$ this can be used to show an error bound of almost optimal order in the maximum norm, see Thomée [18, p. 11].

Remark 2. The restriction $r-\alpha \leq 2$ occurs in (5.8); all other steps of the proof are valid under the less stringent condition $r-\alpha<4$.

For the special case of equation (5.1) with $f=0$, we have the following result.

Corollary 5.3. Let $r=2$ or 3 . Then

$$
\left\|E_{h}(t) P_{h} v-E(t) v\right\| \leq C h^{r} t^{-\frac{r}{4}}\|v\|, \quad t>0, v \in H .
$$

Proof. Lemma 5.2 shows (cf. (5.15))

$$
\left\|E_{h}(t) P_{h} v-E(t) v\right\| \leq C h^{2} t^{-\frac{1}{2}}\|v\|, \quad t>0
$$

which is the desired result when $r=2$. For $r=3$ we note that Lemma 5.2 also shows

$$
\left\|E_{h}(t) P_{h} v-E(t) v\right\| \leq C h^{3}|v|_{3}, \quad t>0,
$$

and the proof can be completed by Helfrich's iteration, cf. Thomée [18, pp. 39-41].

We now turn to the fully discrete case. The backward Euler method applied to (5.3) defines $U_{n} \in \dot{S}_{h}$ by $\left(f_{n}=f\left(t_{n}\right), u_{n}=u\left(t_{n}\right)\right)$

$$
\begin{aligned}
\bar{\partial}_{t} U_{n}+A_{h}^{2} U_{n} & =A_{h} P_{h} f_{n}, \quad t_{n}>0, \\
U_{0} & =P_{h} u_{0} .
\end{aligned}
$$

Analogously to (5.4), we obtain for the difference $e_{n}=U_{n}-u_{n}$ :

$$
G_{h}^{2} \bar{\partial}_{t} e_{n}+e_{n}=\rho_{n}+G_{h} \zeta_{n}+G_{h} \varepsilon_{n}, \quad t_{n}>0,
$$

with

$$
\rho_{n}=\left(R_{h}-I\right) u_{n}, \quad \zeta_{n}=-\left(R_{h}-I\right) G \bar{\partial}_{t} u_{n}, \quad \varepsilon_{n}=-G\left[\bar{\partial}_{t} u_{n}-u_{t}\left(t_{n}\right)\right]
$$

Equation (5.21) is the basis for the estimation of $e_{n}$. It is convenient to first give a lemma providing estimates of $\rho_{n}, \zeta_{n}$, and $\varepsilon_{n}$. In Lemma 5.2 we allowed $\alpha=0$ in order to have Corollary 5.3. In the remainder of this section we assume that $\alpha \geq 1$.

Lemma 5.4. Let $r=2$ or 3 , and let $u$ satisfy (5.1) and (5.2) for some $\alpha \in$ $[1, r]$; assume further that $\alpha \leq \beta \leq r$. Then the following bounds hold for $0<t_{n} \leq T$ :

$$
\begin{aligned}
t_{n-1}\left\|\bar{\partial}_{t} \rho_{n}\right\| & \leq C K h^{\beta} t_{n}^{-\frac{\beta-\alpha}{4}}, \\
\left\|\hat{\rho}_{n}\right\| & \leq C K h^{\beta} t_{n}^{1-\frac{\beta-\alpha}{4}}, \\
\left\|\zeta_{n}\right\| & \leq C K h^{\beta} t_{n}^{-\frac{1}{2}-\frac{\beta-\alpha}{4}}, \\
\left\|\hat{\zeta}_{n}\right\| & \leq C K h^{\beta} t_{n}^{\frac{1}{2}-\frac{\beta-\alpha}{4}}, \\
\left\|\varepsilon_{n}\right\| & \leq C K k t_{n}^{-\frac{1}{2}-\frac{4-\alpha}{4}}, \\
\left\|G_{h} \hat{\varepsilon}_{n}\right\| & \leq C K\left(h^{\beta} t_{n}^{1-\frac{\beta-\alpha}{4}}+k t_{n}^{1-\frac{4-\alpha}{4}}\right),
\end{aligned}
$$


and

$$
\begin{aligned}
& k \sum_{j=1}^{n}\left(t_{j}\left\|\rho_{j}\right\|^{2}+\right. t_{j-1}^{3}\left\|\bar{\partial}_{t} \rho_{j}\right\|^{2}+t_{j}^{-1}\left\|\hat{\rho}_{j}\right\|^{2} \\
&+t_{j}^{2}\left\|\zeta_{j}\right\|^{2}+\left\|\hat{\zeta}_{j}\right\|^{2} \\
&\left.+t_{j}^{2}\left\|\varepsilon_{j}\right\|^{2}+t_{j}^{-1}\left\|G_{h} \hat{\varepsilon}_{j}\right\|^{2}\right) \\
& \leq C K^{2}\left(h^{2 \beta} t_{n}^{2-\frac{\beta-\alpha}{2}}+k^{2} t_{n}^{2-\frac{4-\alpha}{2}}\right),
\end{aligned}
$$

where $\hat{\rho}_{n}=k \sum_{j=1}^{n} \rho_{j}, \hat{\zeta}_{n}=k \sum_{j=1}^{n} \zeta_{j}, \hat{\varepsilon}_{n}=k \sum_{j=1}^{n} \varepsilon_{j}$. Moreover, for $t_{2} \leq$ $t_{n} \leq T$, we have

$$
\begin{aligned}
& \left\|\bar{\partial}_{t} \zeta_{n}\right\| \leq C K h^{\beta} t_{n}^{-\frac{3}{2}-\frac{\beta-\alpha}{4}}, \\
& \left\|\bar{\partial}_{t} \varepsilon_{n}\right\| \leq C K k t_{n}^{-\frac{3}{2}-\frac{4-\alpha}{4}} .
\end{aligned}
$$

Proof. To begin with, (5.5) implies

$$
\left\|\bar{\partial}_{t} \rho_{n}\right\| \leq \frac{1}{k} \int_{t_{n-1}}^{t_{n}}\left\|\rho_{t}\right\| d \tau \leq C K h^{\beta} \frac{1}{k} \int_{t_{n-1}}^{t_{n}} \tau^{-1-\frac{\beta-\alpha}{4}} d \tau \leq C K h^{\beta} t_{n}^{-1-\frac{\beta-\alpha}{4}},
$$

for $n \geq 2$, since $t_{n} \leq 2 t_{n-1}$. This proves (5.22). The bound (5.23) is proved in the same way as (5.7). Next we note that $\hat{\zeta}_{n}=\left(R_{h}-I\right) G\left(u_{n}-u_{0}\right)=\tilde{\eta}_{n}$, and hence $(5.25)$ is the same as (5.8). For the proof of $(5.24)$, we have by $(3.10)$ and (5.2)

$$
\begin{aligned}
\left\|\zeta_{n}\right\| & =\left\|\left(R_{h}-I\right) \frac{1}{k} \int_{t_{n-1}}^{t_{n}} G u_{t} d \tau\right\| \leq C h^{\beta} \frac{1}{k} \int_{t_{n-1}}^{t_{n}}\left\|G u_{t}\right\|_{\beta} d \tau \\
& \leq C K h^{\beta} \frac{1}{k} \int_{t_{n-1}}^{t_{n}} \tau^{-\frac{1}{2}-\frac{\beta-\alpha}{4}} d \tau \leq C K h^{\beta} t_{n-1}^{-\frac{1}{2}-\frac{\beta-\alpha}{4}} \leq C K h^{\beta} t_{n}^{-\frac{1}{2}-\frac{\beta-\alpha}{4}}
\end{aligned}
$$

for $n \geq 2$. For $n=1$, we have instead $\left\|\zeta_{1}\right\|=\frac{1}{k}\left\|\hat{\zeta}_{1}\right\| \leq C K h^{\beta} t_{1}^{-\frac{1}{2}-\frac{\beta-\alpha}{4}}$ by (5.25). In order to prove (5.26), we use Taylor's formula to get

$$
\begin{aligned}
\left\|\varepsilon_{n}\right\| & =\left\|\frac{1}{k} \int_{t_{n-1}}^{t_{n}}\left(\tau-t_{n-1}\right) G u_{t t} d \tau\right\| \leq \int_{t_{n-1}}^{t_{n}}\left\|G u_{t t}\right\| d \tau \\
& \leq C K \int_{t_{n-1}}^{t_{n}} \tau^{-\frac{6-\alpha}{4}} d \tau \leq C K k t_{n-1}^{-\frac{6-\alpha}{4}} \leq C K k t_{n}^{-\frac{1}{2}-\frac{4-\alpha}{4}},
\end{aligned}
$$

for $n \geq 2$, and for $n=1$

$$
\left\|\varepsilon_{1}\right\| \leq \frac{1}{k} \int_{0}^{k} \tau\left\|G u_{t t}\right\| d \tau \leq C K \frac{1}{k} \int_{0}^{k} \tau^{1-\frac{6-\alpha}{4}} d \tau \leq C K k^{1-\frac{6-\alpha}{4}}=C K k t_{1}^{-\frac{1}{2}-\frac{4-\alpha}{4}} .
$$

In a similar way we get

$$
\left\|\bar{\partial}_{t} \zeta_{n}\right\| \leq C h^{\beta} \frac{1}{k} \int_{t_{n-2}}^{t_{n}}\left\|G u_{t t}\right\|_{\beta} d \tau \leq C K h^{\beta} t_{n}^{-\frac{3}{2}-\frac{\beta-\alpha}{4}}
$$

which is (5.29), and

$$
\left\|\bar{\partial}_{t} \varepsilon_{n}\right\| \leq \int_{t_{n-2}}^{t_{n}}\left\|G u_{t t t}\right\| d \tau \leq C K k t_{n}^{-\frac{3}{2}-\frac{4-\alpha}{4}}
$$


where we have used the fact that $\left\|G u_{t t t}(t)\right\| \sim\left\|u_{t t}(t)\right\|_{2} \sim C K t^{-\frac{3}{2}-\frac{4-\alpha}{4}}$, cf. the end of the proof of Theorem 4.1. This proves (5.30). For the estimation of $G_{h} \hat{\varepsilon}_{n}$ we write

$$
\left\|G_{h} \hat{\varepsilon}_{n}\right\| \leq\left\|G \hat{\varepsilon}_{n}\right\|+\left\|\left(G_{h}-G\right) \hat{\varepsilon}_{n}\right\| \text {. }
$$

Here,

$$
\begin{aligned}
\left\|G \hat{\varepsilon}_{n}\right\| & =\left\|k \sum_{j=1}^{n} G \varepsilon_{j}\right\|=\left\|k \sum_{j=1}^{n} \frac{1}{k} \int_{t_{j-1}}^{t_{j}}\left(\tau-t_{j-1}\right) G^{2} u_{t t} d \tau\right\| \\
& \leq k \int_{0}^{t_{n}}\left\|G^{2} u_{t t}\right\| d \tau \leq C K k \int_{0}^{t_{n}} \tau^{-\frac{4-\alpha}{4}} d \tau \leq C K k t_{n}^{1-\frac{4-\alpha}{4}},
\end{aligned}
$$

since $\alpha \geq 1$, and

$$
\begin{aligned}
\left\|\left(G_{h}-G\right) \hat{\varepsilon}_{n}\right\| & =\left\|k \sum_{j=1}^{n}\left(R_{h}-I\right) G \varepsilon_{j}\right\| \leq C h^{\beta}\left\|k \sum_{j=1}^{n} G \varepsilon_{j}\right\|_{\beta} \\
& \leq C h^{\beta}\left(\left\|k \sum_{j=1}^{n} G^{2} \bar{\partial}_{t} u_{j}\right\|_{\beta}+\left\|k \sum_{j=1}^{n} G^{2} u_{t}\left(t_{j}\right)\right\|_{\beta}\right) \\
& \leq C h^{\beta}\left(\int_{0}^{t_{n}}\left\|G^{2} u_{t}\right\|_{\beta} d \tau+k \sum_{j=1}^{n}\left\|G^{2} u_{t}\left(t_{j}\right)\right\|_{\beta}\right) \\
& \leq C K h^{\beta}\left(\int_{0}^{t_{n}} \tau^{-\frac{\beta-\alpha}{4}} d \tau+k \sum_{j=1}^{n} t_{j}^{-\frac{\beta-\alpha}{4}}\right) \leq C K h^{\beta} t_{n}^{1-\frac{\beta-\alpha}{4}},
\end{aligned}
$$

and (5.27) is proved. Finally, (5.28) is an immediate consequence of the previous bounds.

Lemma 5.5. Let $r=2$ or 3 , let $u$ satisfy (5.1) and (5.2) for some $\alpha \in[1, r]$, and let $U_{n}$ be the solution of (5.20). Then

(5.31) $\left\|U_{n}-u\left(t_{n}\right)\right\|_{l} \leq C K\left(h^{r-l} t_{n}^{-\frac{r-\alpha}{4}}+k t_{n}^{-\frac{4+l-\alpha}{4}}\right), \quad 0<t_{n} \leq T, l=0,1$.

Moreover, for the "chemical potential" $w_{n}=A u_{n}-P f_{n}$ and its approximation $W_{n}=A_{h} U_{n}-P_{h} f_{n}$, we have

$$
\left\|W_{n}-w\left(t_{n}\right)\right\| \leq C K\left(h^{r} t_{n}^{-\frac{1}{2}-\frac{r-\alpha}{4}}+k t_{n}^{-\frac{1}{2}-\frac{4-\alpha}{4}}\right), \quad 0<t_{n} \leq T .
$$

Proof. Let $\beta$ be as in Lemma 5.4. In order to prove the case $l=0$ of (5.31), we start out by taking the inner product of (5.21) with $\bar{\partial}_{t} e_{n}$. Using the fact that $G_{h}$ is selfadjoint positive semidefinite on $L_{2}$, we get

$$
\begin{aligned}
\left\|G_{h} \bar{\partial}_{t} e_{n}\right\|^{2}+\left(e_{n}, \bar{\partial}_{t} e_{n}\right) & =\left(\rho_{n}, \bar{\partial}_{t} e_{n}\right)+\left(\zeta_{n}, G_{h} \bar{\partial}_{t} e_{n}\right)+\left(\varepsilon_{n}, G_{h} \bar{\partial}_{t} e_{n}\right) \\
& \leq\left(\rho_{n}, \bar{\partial}_{t} e_{n}\right)+\left\|\zeta_{n}\right\|^{2}+\left\|\varepsilon_{n}\right\|^{2}+\frac{1}{2}\left\|G_{h} \bar{\partial}_{t} e_{n}\right\|^{2},
\end{aligned}
$$

which shows

$$
\left\|G_{h} \bar{\partial}_{t} e_{n}\right\|^{2}+2\left(e_{n}, \bar{\partial}_{t} e_{n}\right) \leq 2\left(\rho_{n}, \bar{\partial}_{t} e_{n}\right)+2\left\|\zeta_{n}\right\|^{2}+2\left\|\varepsilon_{n}\right\|^{2} .
$$

Using the identity

$$
\begin{aligned}
\bar{\partial}_{t}\left(a_{n} b_{n}\right) & =\left(\bar{\partial}_{t} a_{n}\right) b_{n}+a_{n-1}\left(\bar{\partial}_{t} b_{n}\right) \\
& =\left(\bar{\partial}_{t} a_{n}\right) b_{n}+a_{n}\left(\bar{\partial}_{t} b_{n}\right)-k\left(\bar{\partial}_{t} a_{n}\right)\left(\bar{\partial}_{t} b_{n}\right),
\end{aligned}
$$


we obtain

$$
\begin{aligned}
&\left\|G_{h} \bar{\partial}_{t} e_{n}\right\|^{2}+\bar{\partial}_{t}\left\|e_{n}\right\|^{2}+k\left\|\bar{\partial}_{t} e_{n}\right\|^{2} \\
& \leq 2 \bar{\partial}_{t}\left(\rho_{n}, e_{n}\right)-2\left(\bar{\partial}_{t} \rho_{n}, e_{n}\right)+2 k\left(\bar{\partial}_{t} \rho_{n}, \bar{\partial}_{t} e_{n}\right) \\
&+2\left\|\zeta_{n}\right\|^{2}+2\left\|\varepsilon_{n}\right\|^{2} \leq 2 \bar{\partial}_{t}\left(\rho_{n}, e_{n}\right)-2\left(\bar{\partial}_{t} \rho_{n}, e_{n}\right) \\
&+k\left\|\bar{\partial}_{t} \rho_{n}\right\|^{2}+k\left\|\bar{\partial}_{t} e_{n}\right\|^{2}+2\left\|\zeta_{n}\right\|^{2}+2\left\|\varepsilon_{n}\right\|^{2}
\end{aligned}
$$

Cancelling the term $k\left\|\bar{\partial}_{t} e_{n}\right\|^{2}$, multiplying by $t_{n-1}^{2}$ and using (5.33-a) yields

$$
\begin{aligned}
& t_{n-1}^{2}\left\|G_{h} \bar{\partial}_{t} e_{n}\right\|^{2}+\bar{\partial}_{t}\left(t_{n}^{2}\left\|e_{n}\right\|^{2}\right) \\
& \quad \leq 2 t_{n-\frac{1}{2}}\left\|e_{n}\right\|^{2}+2 \bar{\partial}_{t}\left[t_{n}^{2}\left(\rho_{n}, e_{n}\right)\right] \\
& \quad-4 t_{n-\frac{1}{2}}\left(\rho_{n}, e_{n}\right)-2 t_{n-1}^{2}\left(\bar{\partial}_{t} \rho_{n}, e_{n}\right)+t_{n-1}^{3}\left\|\bar{\partial}_{t} \rho_{n}\right\|^{2}+2 t_{n-1}^{2}\left\|\zeta_{n}\right\|^{2}+2 t_{n-1}^{2}\left\|\varepsilon_{n}\right\|^{2} \\
& \leq C\left(\bar{\partial}_{t}\left[t_{n}^{2}\left(\rho_{n}, e_{n}\right)\right]+t_{n}\left\|\rho_{n}\right\|^{2}+t_{n-1}^{3}\left\|\bar{\partial}_{t} \rho_{n}\right\|^{2}+t_{n}^{2}\left\|\zeta_{n}\right\|^{2}+t_{n}^{2}\left\|\varepsilon_{n}\right\|^{2}+t_{n}\left\|e_{n}\right\|^{2}\right) .
\end{aligned}
$$

Multiplying by $k$ and summing with respect to $n$, we obtain after a simple kick-back argument

$$
\begin{aligned}
& k \sum_{j=1}^{n} t_{j-1}^{2}\left\|G_{h} \bar{\partial}_{t} e_{j}\right\|^{2}+t_{n}^{2}\left\|e_{n}\right\|^{2} \\
& \quad \leq C t_{n}^{2}\left\|\rho_{n}\right\|^{2}+C k \sum_{j=1}^{n}\left(t_{j}\left\|\rho_{j}\right\|^{2}+t_{j-1}^{3}\left\|\bar{\partial}_{t} \rho_{j}\right\|^{2}+t_{j}^{2}\left\|\zeta_{j}\right\|^{2}+t_{j}^{2}\left\|\varepsilon_{j}\right\|^{2}+t_{j}\left\|e_{j}\right\|^{2}\right) .
\end{aligned}
$$

Invoking the bounds for $\rho_{n}, \zeta_{n}$ and $\varepsilon_{n}$ in Lemmas 5.1 and 5.4, we conclude that

$$
\begin{aligned}
& k \sum_{j=1}^{n} t_{j-1}^{2}\left\|G_{h} \bar{\partial}_{t} e_{j}\right\|^{2}+t_{n}^{2}\left\|e_{n}\right\|^{2} \\
& \quad \leq C K^{2}\left(h^{2 \beta} t_{n}^{2-\frac{\beta-\alpha}{2}}+k^{2} t_{n}^{2-\frac{4-\alpha}{2}}+k \sum_{j=1}^{n} t_{j}\left\|e_{j}\right\|^{2}\right) .
\end{aligned}
$$

We now have to estimate $k \sum_{j=1}^{n} t_{j}\left\|e_{j}\right\|^{2}$, and we therefore multiply (5.21) by $e_{n}$ to get

$$
\begin{aligned}
\left(G_{h}^{2} \bar{\partial}_{t} e_{n}, e_{n}\right)+\left\|e_{n}\right\|^{2} & =\left(\rho_{n}, e_{n}\right)+\left(\zeta_{n}, G_{h} e_{n}\right)+\left(\varepsilon_{n}, G_{h} e_{n}\right) \\
& \leq \frac{1}{2}\left\|\rho_{n}\right\|^{2}+\frac{1}{2}\left\|e_{n}\right\|^{2}+\left(\left\|\zeta_{n}\right\|+\left\|\varepsilon_{n}\right\|\right)\left\|G_{h} e_{n}\right\|,
\end{aligned}
$$

whence, by (5.33-b),

$$
\bar{\partial}_{t}\left\|G_{h} e_{n}\right\|^{2}+k\left\|G_{h} \bar{\partial}_{t} e_{n}\right\|^{2}+\left\|e_{n}\right\|^{2} \leq\left\|\rho_{n}\right\|^{2}+2\left(\left\|\zeta_{n}\right\|+\left\|\varepsilon_{n}\right\|\right)\left\|G_{h} e_{n}\right\| .
$$

Multiplication by $t_{n}$ and using (5.33-a) now yields

$$
\begin{aligned}
& \bar{\partial}_{t}\left(t_{n+1}\left\|G_{h} e_{n}\right\|^{2}\right)+k t_{n}\left\|G_{h} \bar{\partial}_{t} e_{n}\right\|^{2}+t_{n}\left\|e_{n}\right\|^{2} \\
& \quad \leq\left\|G_{h} e_{n}\right\|^{2}+t_{n}\left\|\rho_{n}\right\|^{2}+2 t_{n}\left(\left\|\zeta_{n}\right\|+\left\|\varepsilon_{n}\right\|\right)\left\|G_{h} e_{n}\right\| \\
& \leq t_{n}\left\|\rho_{n}\right\|^{2}+2 t_{n}^{2}\left\|\zeta_{n}\right\|^{2}+2 t_{n}^{2}\left\|\varepsilon_{n}\right\|^{2}+2\left\|G_{h} e_{n}\right\|^{2}
\end{aligned}
$$


so that, since $G_{h} e_{0}=0$ and in view of (5.28),

$$
\begin{aligned}
k \sum_{j=1}^{n} t_{j}\left\|e_{j}\right\|^{2} & \leq C k \sum_{j=1}^{n}\left(t_{j}\left\|\rho_{j}\right\|^{2}+t_{j}^{2}\left\|\zeta_{j}\right\|^{2}+t_{j}^{2}\left\|\varepsilon_{j}\right\|^{2}+\left\|G_{h} e_{j}\right\|^{2}\right) \\
& \leq C K^{2}\left(h^{2 \beta} t_{n}^{2-\frac{\beta-\alpha}{2}}+k^{2} t_{n}^{2-\frac{4-\alpha}{2}}\right)+2 k \sum_{j=1}^{n}\left\|G_{h} e_{j}\right\|^{2} .
\end{aligned}
$$

To derive an estimate of $k \sum_{j=1}^{n}\left\|G_{h} e_{j}\right\|^{2}$, we sum (5.21) with respect to $n$, taking $G_{h} e_{0}=0$ into account, which yields

$$
G_{h}^{2} e_{n}+\hat{e}_{n}=\hat{\rho}_{n}+G_{h} \hat{\zeta}_{n}+G_{h} \hat{\varepsilon}_{n}, \quad t_{n}>0,
$$

where $\hat{e}_{n}=k \sum_{j=1}^{n} e_{j}, \hat{e}_{0}=0$, etc. Multiplication by $e_{n}=\bar{\partial}_{t} \hat{e}_{n}$ gives

$$
\begin{aligned}
& \left\|G_{h} e_{n}\right\|^{2}+\frac{1}{2} \bar{\partial}_{t}\left\|\hat{e}_{n}\right\|^{2}+\frac{1}{2} k\left\|\bar{\partial}_{t} \hat{e}_{n}\right\|^{2} \\
& \quad=\left(\hat{\rho}_{n}, e_{n}\right)+\left(\hat{\zeta}_{n}, G_{h} e_{n}\right)+\left(G_{h} \hat{\varepsilon}_{n}, e_{n}\right) \\
& \quad \leq \frac{1}{2}\left\|\hat{\zeta}_{n}\right\|^{2}+\frac{1}{2}\left\|G_{h} e_{n}\right\|^{2}+\left(\left\|\hat{\rho}_{n}\right\|+\left\|G_{h} \hat{\varepsilon}_{n}\right\|\right)\left\|e_{n}\right\|,
\end{aligned}
$$

which after some simple manipulation leads to

$$
\begin{gathered}
k \sum_{j=1}^{n}\left\|G_{h} e_{j}\right\|^{2}+\left\|\hat{e}_{n}\right\|^{2} \leq k \sum_{j=1}^{n}\left(\left\|\hat{\zeta}_{j}\right\|^{2}+2\left(\left\|\hat{\rho}_{j}\right\|+\left\|G_{h} \hat{\varepsilon}_{j}\right\|\right)\left\|e_{j}\right\|\right) \\
\leq k \sum_{j=1}^{n}\left(\left\|\hat{\zeta}_{j}\right\|^{2}+2 t_{j}^{-1}\left\|\hat{\rho}_{j}\right\|^{2}+2 t_{j}^{-1}\left\|G_{h} \hat{\varepsilon}_{j}\right\|^{2}+\frac{1}{4} t_{j}\left\|e_{j}\right\|^{2}\right) \\
\quad \leq C K^{2}\left(h^{2 \beta} t_{n}^{2-\frac{\beta-\alpha}{2}}+k^{2} t_{n}^{2-\frac{4-\alpha}{2}}\right)+\frac{1}{4} k \sum_{j=1}^{n} t_{j}\left\|e_{j}\right\|^{2},
\end{gathered}
$$

and, upon substitution into the right-hand side of (5.35),

$$
k \sum_{j=1}^{n} t_{j}\left\|e_{j}\right\|^{2} \leq C K^{2}\left(h^{2 \beta} t_{n}^{2-\frac{\beta-\alpha}{2}}+k^{2} t_{n}^{2-\frac{4-\alpha}{2}}\right) .
$$

Estimates (5.34) and (5.37) now yield

$$
k \sum_{j=1}^{n} t_{j-1}^{2}\left\|G_{h} \bar{\partial}_{t} e_{j}\right\|^{2}+t_{n}^{2}\left\|e_{n}\right\|^{2} \leq C K^{2}\left(h^{2 \beta} t_{n}^{2-\frac{\beta-\alpha}{2}}+k^{2} t_{n}^{2-\frac{4-\alpha}{2}}\right),
$$

and the case $l=0$ of $(5.31)$ follows.

It is now convenient to estimate the difference between $w\left(t_{n}\right)$ and $W_{n}$. Observe that

$$
w\left(t_{n}\right)-W_{n}=-G u_{t}\left(t_{n}\right)+G_{h} \bar{\partial}_{t} U_{n}=G_{h} \bar{\partial}_{t} e_{n}-\zeta_{n}-\varepsilon_{n} .
$$

The last two terms $\zeta_{n}$ and $\varepsilon_{n}$ are estimated as desired by (5.24) and (5.26). In order to estimate $G_{h} \bar{\partial}_{t} e_{n}$, we form the backward difference of (5.21):

$$
G_{h}^{2} \bar{\partial}_{t}^{2} e_{n}+\bar{\partial}_{t} e_{n}=\bar{\partial}_{t} \rho_{n}+G_{h} \bar{\partial}_{t} \zeta_{n}+G_{h} \bar{\partial}_{t} \varepsilon_{n}, \quad n \geq 2
$$

Taking the inner product with $\bar{\partial}_{t} e_{n}$ and using (5.33-b), we get

$$
\begin{aligned}
& \frac{1}{2} \bar{\partial}_{t}\left\|G_{h} \bar{\partial}_{t} e_{n}\right\|^{2}+\frac{1}{2} k\left\|G_{h} \bar{\partial}_{t} e_{n}\right\|^{2}+\left\|\bar{\partial}_{t} e_{n}\right\|^{2} \\
& \quad=\left(\bar{\partial}_{t} \rho_{n}, \bar{\partial}_{t} e_{n}\right)+\left(\bar{\partial}_{t} \zeta_{n}, G_{h} \bar{\partial}_{t} e_{n}\right)+\left(\bar{\partial}_{t} \varepsilon_{n}, G_{h} \bar{\partial}_{t} e_{n}\right) .
\end{aligned}
$$


Hence, by a simple kick-back argument,

$$
\bar{\partial}_{t}\left\|G_{h} \bar{\partial}_{t} e_{n}\right\|^{2} \leq\left\|\bar{\partial}_{t} \rho_{n}\right\|^{2}+2\left(\left\|\bar{\partial}_{t} \zeta_{n}\right\|+\left\|\bar{\partial}_{t} \varepsilon_{n}\right\|\right)\left\|G_{h} \bar{\partial}_{t} e_{n}\right\| .
$$

Multiplying by $t_{n-2}^{3}$, using (5.33) and the fact that $\bar{\partial}_{t}\left(t_{n-1}^{3}\right) \leq 3 t_{n-1}^{2}$, now yields

$$
\begin{aligned}
& \bar{\partial}_{t}\left(t_{n-1}^{3}\left\|G_{h} \bar{\partial}_{t} e_{n}\right\|^{2}\right) \\
& \quad \leq 3 t_{n-1}^{2}\left\|G_{h} \bar{\partial}_{t} e_{n}\right\|^{2}+t_{n-2}^{3}\left\|\bar{\partial}_{t} \rho_{n}\right\|^{2}+2 t_{n-2}^{3}\left(\left\|\bar{\partial}_{t} \zeta_{n}\right\|+\left\|\bar{\partial}_{t} \varepsilon_{n}\right\|\right)\left\|G_{h} \bar{\partial}_{t} e_{n}\right\| \\
& \quad \leq C\left(t_{n-1}^{2}\left\|G_{h} \bar{\partial}_{t} e_{n}\right\|^{2}+t_{n-1}^{3}\left\|\bar{\partial}_{t} \rho_{n}\right\|^{2}+t_{n-2}^{4}\left\|\bar{\partial}_{t} \zeta_{n}\right\|^{2}+t_{n-2}^{4}\left\|\bar{\partial}_{t} \varepsilon_{n}\right\|^{2}\right) .
\end{aligned}
$$

In a standard way we conclude

$$
\begin{aligned}
& t_{n-1}^{3}\left\|G_{h} \bar{\partial}_{t} e_{n}\right\|^{2} \\
& \quad \leq C k \sum_{j=2}^{n}\left(t_{j-1}^{2}\left\|G_{h} \bar{\partial}_{t} e_{j}\right\|^{2}+t_{j-1}^{3}\left\|\bar{\partial}_{t} \rho_{j}\right\|^{2}+t_{j-2}^{4}\left\|\bar{\partial}_{t} \zeta_{j}\right\|^{2}+t_{j-2}^{4}\left\|\bar{\partial}_{t} \varepsilon_{j}\right\|^{2}\right),
\end{aligned}
$$

and hence, by (5.38) and Lemma 5.5,

$$
t_{n-1}^{3}\left\|G_{h} \bar{\partial}_{t} e_{n}\right\|^{2} \leq C K^{2}\left(h^{2 \beta} t_{n}^{2-\frac{\beta-\alpha}{2}}+k^{2} t_{n}^{2-\frac{4-\alpha}{2}}\right),
$$

for $n \geq 2$. For $n=1$ we recall that $G_{h} e_{0}=0$, so that in view of (5.36) and (5.37),

$$
\left\|G_{h} \bar{\partial}_{t} e_{1}\right\|=k^{-1}\left\|G_{h} e_{1}\right\| \leq C K\left(h^{\beta} t_{1}^{-\frac{1}{2}-\frac{\beta-\alpha}{4}}+k t_{1}^{-\frac{1}{2}-\frac{4-\alpha}{4}}\right) .
$$

Taken together, these estimates prove (5.32).

Finally, the estimate of the $H^{1}$ norm of $e_{n}$ is proved by interpolation between the known bounds for the errors in $U_{n}$ and $W_{n}$ just as in the proof of Lemma 5.2.

\section{ERROR BOUNDS FOR THE SEMIDISCRETIZATION IN SPACE}

In this section we shall estimate the difference between the solution $u$ of the nonlinear Cahn-Hilliard equation (2.5) and its semidiscrete approximation $u_{h}$ defined in (3.3). We begin by settling the question of existence, uniqueness and stability for $u_{h}$. Recall the a priori bound

$$
\left\|u_{h}(t)\right\|_{1} \leq C\left(\left\|u_{0 h}\right\|_{1}\right), \quad 0 \leq t<\infty,
$$

that we obtained in (3.4). Since (3.3) is a finite-dimensional system of ordinary differential equations with differentiable nonlinearity, this bound immediately gives global existence:

Lemma 6.1. The initial value problem (3.3) has a unique solution, which exists for all time.

In our error analysis we shall use the following bounds for the nonlinearity $\phi(u)$. 
Lemma 6.2. Let $\|v\|_{1},\|w\|_{1} \leq R$. Then

$$
\begin{aligned}
\left\|\phi^{\prime}(v) z\right\| & \leq C(R)\|z\|_{1}, \\
\|\phi(v)-\phi(w)\| & \leq C(R)\|v-w\|_{1}, \\
\|\phi(v)\| & \leq C(R), \\
\left\|G_{h}^{\frac{1}{2}}\left[\phi^{\prime}(v) z\right]\right\| & \leq C(R)\|z\|, \\
\left\|G_{h}^{\frac{1}{2}}[\phi(v)-\phi(w)]\right\| & \leq C(R)\|v-w\|, \\
\left\|G_{h}^{\frac{1}{2}}\left(\left[\phi^{\prime}(v)-\phi^{\prime}(w)\right] z\right)\right\| & \leq C(R)\|v-w\|\|z\|_{1} .
\end{aligned}
$$

Proof. We only demonstrate (6.5) and (6.6); the remaining bounds are proved in a similar way. First note that, by Hölder's and Sobolev's inequalities $(d \leq 3)$,

$$
\left\|G_{h}^{\frac{1}{2}} f\right\|=\sup _{\chi \in S_{h}} \frac{|(f, \chi)|}{|\chi|_{1}} \leq C\|f\|_{L_{6 / 5}} .
$$

Since by assumption (2.4), $\phi$ is a cubic polynomial if $d=3$, we thus have

$$
\begin{aligned}
\left\|G_{h}^{\frac{1}{2}}\left[\phi^{\prime}(v) z\right]\right\| & \leq C\left\|\phi^{\prime}(v) z\right\|_{L_{6 / 5}} \leq C\left\|\phi^{\prime}(v)\right\|_{L_{3}}\|z\|_{L_{2}} \\
& \leq C\left(1+\|v\|_{L_{6}}^{2}\right)\|z\| \leq C\left(1+\|v\|_{1}^{2}\right)\|z\|,
\end{aligned}
$$

which is (6.5), and (6.6) readily follows. The modification needed when $d \leq 2$ and $\phi$ has arbitrary degree is obvious.

Remark. The local Lipschitz condition (6.6) was used by Thomée and Wahlbin [19] in the error analysis of finite element methods for semilinear parabolic problems of second order.

We also need the following well-known generalization of Gronwall's lemma. We include a proof for the sake of completeness.

Lemma 6.3. Let the function $\varphi(t, \tau) \geq 0$ be continuous for $0 \leq \tau<t \leq T$. If

$$
\varphi(t, \tau) \leq A(t-\tau)^{-1+\alpha}+B \int_{\tau}^{t}(t-s)^{-1+\beta} \varphi(s, \tau) d s, \quad 0 \leq \tau<t \leq T,
$$

for some constants $A, B \geq 0, \alpha, \beta>0$, then there is a constant $C=$ $C(B, T, \alpha, \beta)$ such that

$$
\varphi(t, \tau) \leq C A(t-\tau)^{-1+\alpha}, \quad 0 \leq \tau<t \leq T .
$$

Proof. Iterating the given inequality $N-1$ times, using the identity

$$
\int_{\tau}^{t}(t-s)^{-1+\alpha}(s-\tau)^{-1+\beta} d s=C(\alpha, \beta)(t-\tau)^{-1+\alpha+\beta}, \quad \alpha, \beta>0,
$$

and estimating $(t-\tau)^{\beta}$ by $T^{\beta}$, we obtain

$$
\varphi(t, \tau) \leq C_{1} A(t-\tau)^{-1+\alpha}+C_{2} \int_{\tau}^{t}(t-s)^{-1+N \beta} \varphi(s, \tau) d s, \quad 0 \leq \tau<t \leq T,
$$

where $C_{1}=C_{1}(B, T, \alpha, \beta, N), C_{2}=C_{2}(B, \beta, N)$. We now choose the smallest $N$ such that $-1+N \beta \geq 0$, and estimate $(t-s)^{-1+N \beta}$ by $T^{-1+N \beta}$. If $-1+\alpha \geq 0$, we obtain the desired conclusion by the standard version of Gronwall's lemma. Otherwise, we set $\psi(t, \tau)=(t-\tau)^{1-\alpha} \varphi(t, \tau)$ to obtain

$$
\psi(t, \tau) \leq C_{1} A+C_{3} \int_{\tau}^{t}(s-\tau)^{-1+\alpha} \psi(s, \tau) d s, \quad 0 \leq \tau<t \leq T,
$$


and the standard version of Gronwall's lemma yields $\psi(t, \tau) \leq C A$ for $0 \leq$ $\tau<t \leq T$, which is the desired result.

We now turn to the stability of $u_{h}$ with respect to perturbations of the initial value.

Lemma 6.4. Let $u_{h}^{(i)}, i=1,2$, be two solutions of (3.3) with initial values $u_{0 h}^{(i)}$ and satisfying $\left\|u_{h}^{(i)}(t)\right\|_{1} \leq R$ for $0 \leq t \leq T, i=1,2$. Then for $j=$ $-1,0,1, j \leq l=0,1$ we have

$$
\left|u_{h}^{(1)}(t)-u_{h}^{(2)}(t)\right|_{l} \leq C(R, T) t^{-\frac{l-j}{4}}\left|u_{0 h}^{(1)}-u_{0 h}^{(2)}\right|_{j}, \quad 0<t \leq T .
$$

Proof. The proof is more or less the same as that of Theorem 6.5 and we omit it.

We are now ready to formulate our main result.

Theorem 6.5. Let $r=2$ or 3 , and assume that for some $\alpha \in[1, r]$ we have $u_{0} \in \dot{H}^{\alpha}$ with

$$
\left\|u_{0}\right\|_{\alpha} \leq R_{1} ; \quad\|u(t)\|_{1}+\left\|u_{h}(t)\right\|_{1} \leq R_{2}, \quad 0 \leq t \leq T,
$$

where $u$ and $u_{h}$ are the solutions of (2.5) and (3.3), respectively. Then

$$
\begin{aligned}
& \text { 1) }\left\|u_{h}(t)-u(t)\right\| \leq C\left\|u_{0 h}-P_{h} u_{0}\right\|+C h^{r} t^{-\frac{r-\alpha}{4}}, \\
& \text { 2) }\left\|u_{h}(t)-u(t)\right\|_{1} \leq C t^{-\frac{1-j}{4}}\left|u_{0 h}-P_{h} u_{0}\right|_{j}+C h^{r-1} t^{-\frac{r-\alpha}{4}}, \quad j=0,1,
\end{aligned}
$$

for $0<t \leq T$, where $C=C\left(R_{1}, R_{2}, T\right)$.

Proof. It follows from Lemma 6.4 that we may assume that $u_{0 h}=P_{h} u_{0}$; otherwise, the additional errors in (6.11) and (6.12) caused by such a perturbation of the discrete initial value are bounded by

$$
C\left\|u_{0 h}-P_{h} u_{0}\right\| \text { and } C t^{-\frac{1-j}{4}}\left|u_{0 h}-P_{h} u_{0}\right|_{j}, \quad j=-1,0,1,0<t \leq T,
$$

respectively. Assuming thus that $u_{0 h}=P_{h} u_{0}$, we shall compare $u_{h}$ with the auxiliary function $\tilde{u}_{h}(t) \in \dot{S}_{h}$ defined by

$$
\begin{aligned}
\tilde{u}_{h, t}+A_{h}^{2} \tilde{u}_{h} & =-A_{h} P_{h} \phi(u), \quad t>0, \\
\tilde{u}_{h}(0) & =P_{h} u_{0} .
\end{aligned}
$$

Setting $e=u_{h}-u$ and $\tilde{e}=\tilde{u}_{h}-u$, we know from Lemma 5.2 and (6.10) that

$$
\|\tilde{e}(t)\|_{l} \leq C\left(R_{1}, T\right) h^{r-l} t^{-\frac{r-\alpha}{4}}, \quad 0<t \leq T, l=0,1 .
$$

By Duhamel's principle (3.5), we have

$$
\begin{aligned}
e(t) & =\tilde{e}(t)+\left(u_{h}(t)-\tilde{u}_{h}(t)\right) \\
& =\tilde{e}(t)-\int_{0}^{t} E_{h}(t-\tau) A_{h} P_{h}\left[\phi\left(u_{h}(\tau)\right)-\phi(u(\tau))\right] d \tau \\
& =\tilde{e}(t)-\int_{0}^{t} A_{h}^{\frac{3}{2}} E_{h}(t-\tau) G_{h}^{\frac{1}{2}}\left[\phi\left(u_{h}(\tau)\right)-\phi(u(\tau))\right] d \tau .
\end{aligned}
$$

By (6.14), the Lipschitz condition (6.6) and (6.10), we obtain

$$
\|e(t)\| \leq C\left(R_{1}, T\right) h^{r} t^{-\frac{r-\alpha}{4}}+C\left(R_{2}\right) \int_{0}^{t}(t-\tau)^{-\frac{3}{4}}\|e(\tau)\| d \tau,
$$


and Gronwall's Lemma 6.3 shows

$$
\|e(t)\| \leq C\left(R_{1}, R_{2}, T\right) h^{r} t^{-\frac{r-\alpha}{4}}, \quad 0<t<T .
$$

For the proof of (6.12), we use (6.3) instead:

$$
\begin{aligned}
\|e(t)\|_{1} & \leq\|\tilde{e}(t)\|_{1}+C \int_{0}^{t}\left\|A_{h}^{\frac{3}{2}} E_{h}(t-\tau) P_{h}\left[\phi\left(u_{h}(\tau)\right)-\phi(u(\tau))\right]\right\| d \tau \\
& \leq C\left(R_{1}, T\right) h^{r-1} t^{-\frac{r-\alpha}{4}}+C\left(R_{2}\right) \int_{0}^{t}(t-\tau)^{-\frac{3}{4}}\|e(\tau)\|_{1} d \tau,
\end{aligned}
$$

and (6.12) follows by Gronwall's lemma.

In order to apply the above result, we must verify assumption (6.10). In view of (2.8) and (6.1), we find that (6.10) holds, for example, if it can be proved that $\left\|u_{0 h}\right\|_{1} \leq C\left\|u_{0}\right\|_{1}$ independently of $h$. Clearly, this holds if $u_{0 h}=R_{h} u_{0}$. Another possibility is to choose $u_{0 h}=P_{h} u_{0}$, provided that we have the inverse inequality

$$
\|\chi\|_{1} \leq C h^{-1}\|\chi\| \quad \forall \chi \in \dot{S}_{h} .
$$

It is easy to see that (6.15) and (3.10) imply $\left\|P_{h} u_{0}\right\|_{1} \leq C\left\|u_{0}\right\|_{1}$.

In view of (3.10) and (3.11), we have

$$
\left|R_{h} u_{0}-P_{h} u_{0}\right|_{j} \leq\left|R_{h} u_{0}-u_{0}\right|_{j}+\left|u_{0}-P_{h} u_{0}\right|_{j} \leq C h^{\beta-j}\left|u_{0}\right|_{\beta},
$$

for $1 \leq \beta \leq r$ and $j=0$ if $r=2, j=-1,0$ if $r=3$. (The negative norm bound for the error in $P_{h}$ follows from (3.11) by a well-known duality argument.) The following corollaries are now evident.

Corollary 6.6 (Smooth data). Let $r=2$ or 3 , and assume that $u_{0} \in \dot{H}^{r}$ with $\left|u_{0}\right|_{r} \leq R$.

(1) If $u_{0 h}=R_{h} u_{0}$, then

$$
\left\|u_{h}(t)-u(t)\right\| \leq C(R, T) h^{r}, \quad 0 \leq t \leq T ;
$$

(2) if $u_{0 h}=P_{h} u_{0}$ and (6.15) holds, then

$$
\left\|u_{h}(t)-u(t)\right\|_{l} \leq C(R, T) h^{r-l}, \quad 0 \leq t \leq T, l=0,1 .
$$

Corollary 6.7 (Data in $H^{1}$ ). Let $r=2$ or 3 , and assume that $u_{0} \in \dot{H}^{1}$ with $\left|u_{0}\right|_{1} \leq R$.

(1) If $u_{0 h}=R_{h} u_{0}$, then

$$
\left\|u_{h}(t)-u(t)\right\|_{1} \leq C(R, T) h^{r-1} t^{-\frac{r-1}{4}}, \quad 0<t \leq T
$$

(2) if $u_{0 h}=P_{h} u_{0}$ and (6.15) holds, then

$$
\left\|u_{h}(t)-u(t)\right\|_{l} \leq C(R, T) h^{r-l} t^{-\frac{r-1}{4}}, \quad 0<t \leq T, l=0,1 .
$$

The estimation of the error in the semidiscrete "chemical potential" $w_{h}=$ $A_{h} u_{h}+P_{h} \phi\left(u_{h}\right)$ is more technical. We shall only present a result for the case of nonsmooth data: $u_{0} \in \dot{H}^{1}$. In the proof of this we shall need the following bound for $u_{h, t}$. 
Lemma 6.8. Let $\left\|u_{0 h}\right\|_{1} \leq R$. Then

$$
\left\|u_{h, t}(t)\right\|_{l} \leq C(R, T) t^{-1-\frac{l-1}{4}}, \quad 0<t \leq T, l=0,1 .
$$

Proof. Let $z_{h}=u_{h, t}$. Then by differentiation of (3.3), we have

$$
z_{h, t}+A_{h}^{2} z_{h}=-A_{h} P_{h} \phi^{\prime}\left(u_{h}\right) z_{h},
$$

and after multiplication by $t$,

$$
\begin{aligned}
D_{t}\left(t z_{h}\right)+A_{h}^{2}\left(t z_{h}\right) & =z_{h}-t A_{h} P_{h} \phi^{\prime}\left(u_{h}\right) z_{h} \\
& =-A_{h}^{2} u_{h}-A_{h} P_{h} \phi\left(u_{h}\right)-t A_{h} P_{h} \phi^{\prime}\left(u_{h}\right) z_{h},
\end{aligned}
$$

where we have used (3.3) in the last step. Hence,

$$
t z_{h}(t)=-\int_{0}^{t} E_{h}(t-s)\left(A_{h}^{2} u_{h}(s)+A_{h} P_{h} \phi\left(u_{h}(s)\right)+s A_{h} P_{h} \phi^{\prime}\left(u_{h}(s)\right) z_{h}(s)\right) d s,
$$

so that, by the boundedness of $\left\|u_{h}\right\|_{1},(6.4)$ and (6.5),

$$
\begin{aligned}
t\left\|z_{h}(t)\right\| & \leq C \int_{0}^{t}(t-s)^{-\frac{3}{4}}\left(\left\|u_{h}(s)\right\|_{1}+\left\|G_{h}^{\frac{1}{2}} \phi\left(u_{h}(s)\right)\right\|+s\left\|G_{h}^{\frac{1}{2}}\left[\phi^{\prime}\left(u_{h}(s)\right) z_{h}(s)\right]\right\|\right) d s \\
& \leq C(R) t^{\frac{1}{4}}+C(R) \int_{0}^{t}(t-s)^{-\frac{3}{4}} s\left\|z_{h}(s)\right\| d s .
\end{aligned}
$$

Now Gronwall's Lemma 6.3 yields $t\left\|z_{h}(t)\right\| \leq C(R, T) t^{\frac{1}{4}}$ for $0<t \leq T$, which proves the case $l=0$ of the lemma. The proof for the case $l=1$ can be based on the first identity in (6.17). We proceed in the same way, using the known bound for $\left\|z_{h}\right\|$ and the bound (6.2) for $\left\|\phi^{\prime}\left(u_{h}\right) z_{h}\right\|$.

Theorem 6.9. Let $r=2$ or 3 and let $u_{0} \in \dot{H}^{1}$ with $\left|u_{0}\right|_{1} \leq R$, and $u_{0 h}=P_{h} u_{0}$. Then for the "chemical potential" $w=A u+P \phi(u)$ and its approximation $w_{h}=$ $A_{h} u_{h}+P_{h} \phi\left(u_{h}\right)$ we have

$$
\left\|w_{h}(t)-w(t)\right\| \leq C(R, T) h^{r} t^{-\frac{1}{2}-\frac{r-1}{4}}, \quad 0<t \leq T .
$$

Proof. Again, we use the auxiliary function $\tilde{u}_{h}$ defined in (6.13). Let $e=$ $u_{h}-u, z_{h}=u_{h}-\tilde{u}_{h}$, and $\tilde{w}_{h}=A_{h} \tilde{u}_{h}+P_{h} \phi(u)=-G_{h} \tilde{u}_{h, t}$. Since $w_{h}-w=$ $\left(w_{h}-\tilde{w}_{h}\right)+\left(\tilde{w}_{h}-w\right)$, where by Lemma 5.2

$$
\left\|\tilde{w}_{h}(t)-w(t)\right\| \leq C h^{r} t^{-\frac{1}{2}-\frac{r-1}{4}}=C h^{r} t^{-\frac{r+1}{4}}, \quad 0<t \leq T,
$$

it remains to estimate $w_{h}-\tilde{w}_{h}=-G_{h}\left(u_{h, t}-\tilde{u}_{h, t}\right)=-G_{h} z_{h, t}$. The function $z_{h}$ satisfies

$$
z_{h, t}+A_{h}^{2} z_{h}=A_{h} P_{h} f, \quad f=\phi(u)-\phi\left(u_{h}\right) .
$$

Differentiating this equation we get

$$
z_{h, t t}+A_{h}^{2} z_{h, t}=A_{h} P_{h} f_{t} .
$$

Multiplying by $t$ and using (6.19) yields

$$
D_{t}\left(t z_{h, t}\right)+A_{h}^{2}\left(t z_{h, t}\right)=z_{h, t}+t A_{h} P_{h} f_{t}=-A_{h}^{2} z_{h}+A_{h} P_{h} f+t A_{h} P_{h} f_{t} .
$$

Hence, by Duhamel's principle,

$$
\begin{aligned}
t G_{h} z_{h, t}(t)= & -\int_{0}^{t} A_{h} E_{h}(t-s) z_{h}(s) d s+\int_{0}^{t} A_{h}^{\frac{1}{2}} E_{h}(t-s) G_{h}^{\frac{1}{2}} f(s) d s \\
& +\int_{0}^{t} E_{h}(t-s) P_{h} f_{t}(s) s d s \equiv I_{1}+I_{2}+I_{3} .
\end{aligned}
$$


Here, by the error bounds in Lemma 5.2 and Theorem 6.5, we have

$$
\left\|I_{1}\right\| \leq C \int_{0}^{t}(t-s)^{-\frac{1}{2}}\left\|z_{h}(s)\right\| d s \leq C h^{r} \int_{0}^{t}(t-s)^{-\frac{1}{2}} s^{-\frac{r-1}{4}} d s \leq C h^{r} t^{1-\frac{r+1}{4}} .
$$

Similarly, by (6.6),

$$
\left\|I_{2}\right\| \leq C \int_{0}^{t}(t-s)^{-\frac{1}{4}}\|e(s)\| d s \leq C h^{r} t^{1-\frac{r}{4}} .
$$

For $I_{3}$, we write $f_{t}=-\left[\phi^{\prime}\left(u_{h}\right)-\phi^{\prime}(u)\right] u_{h, t}-\phi^{\prime}(u) e_{t}$, so that

$$
\begin{aligned}
I_{3}= & -\int_{0}^{t} A_{h}^{\frac{1}{2}} E_{h}(t-s) G_{h}^{\frac{1}{2}}\left(\left[\phi^{\prime}\left(u_{h}(s)\right)-\phi^{\prime}(u(s))\right] u_{h, t}(s)\right) s d s \\
& -\int_{0}^{t}\left[E_{h}(t-s) P_{h}-E(t-s)\right]\left[\phi^{\prime}(u(s)) e_{t}(s)\right] s d s \\
& -\int_{0}^{t} E(t-s)\left[\phi^{\prime}(u(s)) e_{t}(s)\right] s d s \equiv I_{4}+I_{5}+I_{6} .
\end{aligned}
$$

Here, by (6.7), Theorem 6.5 and Lemma 6.8 , we have

$$
\left\|I_{4}\right\| \leq C \int_{0}^{t}(t-s)^{-\frac{1}{4}}\left\|u_{h, t}(s)\right\|_{1}\|e(s)\| s d s \leq C h^{r} t^{1-\frac{r}{4}} .
$$

Further, by Corollary 5.3, (6.2) and the bounds for $u_{t}$ and $u_{h, t}$ in Theorem 4.1 and Lemma 6.8 , we obtain

$$
\left\|I_{5}\right\| \leq C h^{r} \int_{0}^{t}(t-s)^{-\frac{r}{4}}\left\|e_{t}(s)\right\|_{1} s d s \leq C h^{r} t^{1-\frac{r}{4}} .
$$

For $I_{6}$ we argue as follows. Let $\chi \in L_{2}$ be arbitrary. Then

$$
\begin{aligned}
\left(E(t-s)\left[\phi^{\prime}(u(s)) e_{t}(s)\right], \chi\right) & =\left(G e_{t}(s), A P\left[\phi^{\prime}(u(s)) E(t-s) \chi\right]\right) \\
& \leq\left\|G e_{t}(s)\right\|\left\|A P\left[\phi^{\prime}(u(s)) E(t-s) \chi\right]\right\| .
\end{aligned}
$$

By a careful exploitation of Sobolev's inequality $(d \leq 3)$ and the moment inequality

$$
|v|_{\beta} \leq C|v|_{\delta}^{1-\theta}|v|_{\gamma}^{\theta} \quad \beta=(1-\theta) \delta+\theta \gamma, \theta \in[0,1],
$$

we may show

$$
\left\|A P\left[\phi^{\prime}(u) v\right]\right\| \leq C\left(1+|u|_{i}|u|_{\gamma}\right)|v|_{2},
$$

where $\frac{5}{2}<\gamma<3$ (cf. the proof of Lemma A.1 in the supplement). Hence, by the regularity estimates for $u(t)$ and $E(t)$, we have

$$
\begin{aligned}
\left\|A\left[\phi^{\prime}(u(s)) E(t-s) \chi\right]\right\| & \leq C\left(1+|u(s)|_{1}|u(s)|_{\gamma}\right)|E(t-s) \chi|_{2} \\
& \leq C(t-s)^{-\frac{1}{2}} s^{-\frac{\chi-1}{4}}\|\chi\|,
\end{aligned}
$$

and, since $\chi$ is arbitrary, we conclude that

$$
\left\|E(t-s)\left[\phi^{\prime}(u(s)) e_{t}(s)\right]\right\| \leq C(t-s)^{-\frac{1}{2}} s^{-\frac{\sigma}{4}}\left\|G e_{t}(s)\right\|,
$$

where $\sigma=\gamma-1 \in\left(\frac{3}{2}, 2\right)$. Therefore,

$$
\left\|I_{6}\right\| \leq C \int_{0}^{t}(t-s)^{-\frac{1}{2}} s^{1-\frac{\sigma}{4}}\left\|G e_{t}(s)\right\| d s .
$$


Here, $G e_{t}=-\left(G_{h}-G\right) u_{h, t}-\left(\tilde{w}_{h}-w\right)+G_{h} z_{h, t}$, where by (3.10) and Lemma 6.8 with $l=r-2$ (hence $l=0$ or 1 ),

$$
\left\|\left(G_{h}-G\right) u_{h, t}(t)\right\| \leq C h^{r}\left\|G u_{h, t}(t)\right\| \leq C h^{r}\left\|u_{h, t}(t)\right\|_{r-2} \leq C h^{r} t^{-\frac{r+1}{4}} .
$$

Taking this together with (6.18) and the above bounds for $I_{j}, j=1, \ldots, 6$, we now have

$$
t G_{h} z_{h, t}(t) \leq C h^{r} t^{1-\frac{r+1}{4}}+C \int_{0}^{t}(t-s)^{-\frac{1}{2}} s^{1-\frac{\sigma}{4}}\left\|G_{h} z_{h, t}(s)\right\| d s, \quad 0<t \leq T,
$$

or, with $\varphi(t)=t G_{h} z_{h, t}(t)$,

$$
\varphi(t) \leq C h^{r} t^{1-\frac{r+1}{4}}+C \int_{0}^{t}(t-s)^{-\frac{1}{2}} s^{-\frac{\sigma}{4}} \varphi(s) d s, \quad 0<t \leq T .
$$

Iterating this inequality once, recalling that $\sigma<2$, we obtain (cf. the proof of the Gronwall Lemma 6.3)

$$
\varphi(t) \leq C h^{r} t^{1-\frac{r+1}{4}}+C \int_{0}^{t} s^{-\frac{\sigma}{2}} \varphi(s) d s, \quad 0<t \leq T,
$$

and since $1-(r+1) / 4 \geq 0$ the standard Gronwall lemma shows $\varphi(t) \leq$ $C h^{r} t^{1-\frac{r+1}{4}}$ for $0<t \leq T$, which implies the desired bound for $G_{h} z_{h, t}$.

\section{ERROR BOUNDS FOR THE COMPLETELY DISCRETE SCHEME}

The purpose of this section is to estimate the difference between the solution $u$ of the Cahn-Hilliard equation (2.5) and its completely discrete approximation $U_{n}$ defined in (3.6). The argument is completely parallel to that of the previous section and we only present an outline indicating the modifications needed. We first recall that, if $k \leq 4 / \beta^{4}$, then we have the a priori bound (3.8). Using this bound, we conclude that (3.6) has a unique solution $U_{n}$ for all $t_{n}$ if $k$ is small.

In the proof of our main result we need a discrete version of the Gronwall Lemma 6.3:

Lemma 7.1. Let $0 \leq \varphi_{n} \leq R$ for $0 \leq t_{n} \leq T$. If

$$
\varphi_{n} \leq A_{1} t_{n}^{-1+\alpha_{1}}+A_{2} t_{n}^{-1+\alpha_{2}}+B k \sum_{j=1}^{n} t_{n-j+1}^{-1+\beta} \varphi_{j}, \quad 0<t_{n} \leq T,
$$

for some constants $A_{1}, A_{2}, B \geq 0, \alpha_{1}, \alpha_{2}, \beta>0$, then there are constants $k_{0}=k_{0}(R, B, \beta)$ and $C=C\left(B, T, \alpha_{1}, \alpha_{2}, \beta\right)$ such that, for $k \leq k_{0}$,

$$
\varphi_{n} \leq C\left(A_{1} t_{n}^{-1+\alpha_{1}}+A_{2} t_{n}^{-1+\alpha_{2}}\right), \quad 0<t_{n} \leq T .
$$

Proof. The proof is completely analogous to the proof of Lemma 6.3. Iterating the given inequality, using

$$
k \sum_{j=1}^{n} t_{n-j+1}^{-1+\alpha} t_{j}^{-1+\beta} \leq C(\alpha, \beta) t_{n}^{-1+\alpha+\beta}, \quad \alpha, \beta>0,
$$


which follows by comparison with the integral in (6.9), we get

$$
\begin{aligned}
\varphi_{n} \leq & C_{1}\left(A_{1} t_{n}^{-1+\alpha_{1}}+A_{2} t_{n}^{-1+\alpha_{2}}\right)+C_{2} k \sum_{j=1}^{n} t_{n-j+1}^{-1+N \beta} \varphi_{j} \\
\leq & C_{1}\left(A_{1} t_{n}^{-1+\alpha_{1}}+A_{2} t_{n}^{-1+\alpha_{2}}\right) \\
& +C_{2} k \sum_{j=1}^{n-1} t_{n-j+1}^{-1+N \beta} \varphi_{j}+C_{2} k^{N \beta} R, \quad 0<t_{n} \leq T,
\end{aligned}
$$

where $C_{1}$ and $C_{2}$ are the same as before, and $-1+N \beta \geq 0$. If $k$ is small, then we may cancel the last term on the right and the proof is completed by means of the standard discrete Gronwall lemma. In this connection, if $\alpha_{1} \geq \alpha_{2}$, say, and $-1+\alpha_{2}<0$, we first set $\psi_{n}=t_{n}^{1-\alpha_{2}} \varphi_{n}$ to get

$$
\psi_{n} \leq C_{3}\left(A_{1} t_{n}^{\alpha_{1}-\alpha_{2}}+A_{2}\right)+C_{4} k \sum_{j=1}^{n-1} t_{j}^{-1+\alpha_{2}} \psi_{j}, \quad 0<t_{n} \leq T,
$$

which leads to $\psi_{n} \leq C\left(A_{1} t_{n}^{\alpha_{1}-\alpha_{2}}+A_{2}\right)$ for $0<t_{n} \leq T$.

We can now state our main result. For simplicity of presentation we assume that $u_{0 h}=P_{h} u_{0}$. The modifications needed for other choices of discrete initial data are exactly the same as in the previous section.

Theorem 7.2. Let $r=2$ or 3 , and assume that for some $\alpha \in[1, r]$ we have $u_{0} \in \dot{H}^{\alpha}$ and $u_{0 h}=P_{h} u_{0}$ with

$$
\left\|u_{0}\right\|_{\alpha} \leq R_{1} ; \quad\left\|u\left(t_{n}\right)\right\|_{1}+\left\|U_{n}\right\|_{1} \leq R_{2}, \quad 0 \leq t_{n} \leq T,
$$

where $u$ and $U_{n}$ are the solutions of (2.5) and (3.6), respectively. Then there are $k_{0}=k_{0}\left(R_{2}\right)$ and $C=C\left(R_{1}, R_{2}, T\right)$ such that, for $k \leq k_{0}$,

$$
\left\|U_{n}-u\left(t_{n}\right)\right\|_{l} \leq C\left(h^{r-l} t_{n}^{-\frac{r-\alpha}{4}}+k t_{n}^{-\frac{4+l-\alpha}{4}}\right), \quad 0<t_{n} \leq T, l=0,1 .
$$

Proof. We define $\widetilde{U}_{n} \in \dot{S}_{h}$ by

$$
\begin{aligned}
& \bar{\partial}_{t} \widetilde{U}_{n}+A_{h}^{2} \widetilde{U}_{n}=-A_{h} P_{h} \phi\left(u\left(t_{n}\right)\right), \quad t_{n}>0, \\
& \widetilde{U}_{0}=P_{h} u_{0} .
\end{aligned}
$$

With $e_{n}=\left[U_{n}-\tilde{U}_{n}\right]+\left[\tilde{U}_{n}-u\left(t_{n}\right)\right] \equiv Z_{n}+\tilde{e}_{n}$, we know from Lemma 5.5 that

$$
\left\|\tilde{e}_{n}\right\|_{l} \leq C\left(R_{1}, T\right)\left(h^{r-l} t_{n}^{-\frac{r-\alpha}{4}}+k t_{n}^{-\frac{4+l-\alpha}{4}}\right), \quad 0<t_{n} \leq T, l=0,1 .
$$

We first demonstrate the case $l=0$. By the variation of constants formula (3.9) we have

$$
e_{n}=\tilde{e}_{n}-k \sum_{j=1}^{n} E_{k h}^{n-j+1} A_{h} P_{h}\left[\phi\left(U_{j}\right)-\phi\left(u\left(t_{j}\right)\right)\right] .
$$

Using the fact that

$$
\left\|A_{h}^{\frac{\beta}{2}} E_{k h}^{n} v\right\| \leq C_{\beta} t_{n}^{-\frac{\beta}{4}}\|v\|, \quad t_{n}>0, \quad \beta \geq 0
$$

and the Lipschitz condition (6.6), we obtain

$$
\left\|e_{n}\right\| \leq C\left(R_{1}, T\right)\left(h^{r} t_{n}^{-\frac{r-\alpha}{4}}+k t_{n}^{-\frac{4-\alpha}{4}}\right)+C\left(R_{2}\right) k \sum_{j=1}^{n} t_{n-j+1}^{-\frac{3}{4}}\left\|e_{j}\right\|, \quad 0<t_{n} \leq T,
$$


and the desired bound follows by the discrete Gronwall Lemma 7.1. Similarly, for the case $l=1$ we use the discrete analogue of the proof of (6.12). However, this does not work when $\alpha=1$, owing to the strength of the singularity of the term $k t_{n}^{-\frac{4+l-\alpha}{4}}$ in (7.1). Instead, we argue as follows when $\alpha=1$ : From the equation for $Z_{n}$ and (5.33) it follows that

$$
\bar{\partial}_{t}\left(t_{n} Z_{n}\right)+A_{h}^{2}\left(t_{n} Z_{n}\right)=Z_{n-1}-t_{n} A_{h} P_{h}\left[\phi\left(U_{n}\right)-\phi\left(u\left(t_{n}\right)\right)\right], \quad t_{n}>0 .
$$

Using $Z_{0}=0$ and (6.3), we obtain by the variation of constants formula

$$
t_{n}\left\|Z_{n}\right\|_{1} \leq k \sum_{j=2}^{n}\left\|A_{h}^{\frac{1}{2}} E_{k h}^{n-j+1}\right\|\left\|Z_{j-1}\right\|+C\left(R_{2}\right) k \sum_{j=1}^{n}\left\|A_{h}^{\frac{3}{2}} E_{k h}^{n-j+1}\right\| t_{j}\left\|e_{j}\right\|_{1} .
$$

By a modification of the first part of this proof we have here (with $\alpha=1$ )

$$
\left\|Z_{j}\right\| \leq C\left(R_{1}, R_{2}, T\right)\left(h^{r-1} t_{j}^{-\frac{r-2}{4}}+k t_{j}^{-\frac{3}{4}}\right), \quad 0<t_{j} \leq T .
$$

Together with (7.1), this shows

$$
\begin{aligned}
t_{n}\left\|e_{n}\right\|_{1} \leq & C\left(R_{1}, R_{2}, T\right)\left(h^{r-1} t_{n}^{1-\frac{r-1}{4}}+k\right) \\
& +C\left(R_{2}\right) k \sum_{j=1}^{n} t_{n-j+1}^{-\frac{3}{4}} t_{j}\left\|e_{j}\right\|_{1}, \quad 0<t_{n} \leq T,
\end{aligned}
$$

and the desired result follows.

\section{StABILITY OF ATtRACTORS}

Let $u(t)=\mathscr{T}(t)\left(u_{0}\right)$ denote the solution of the Cahn-Hilliard equation (2.5). Then $\mathscr{T}(t)$ is a nonlinear semigroup in $\dot{H}^{1}$. Similarly, (3.3) and (3.6) define nonlinear semigroups $\mathscr{T}_{h}(t)$ and $\mathscr{T}_{h k}^{n}$ in $\dot{S}_{h} \subset \dot{H}^{1}$ by $u_{h}(t)=\mathscr{T}_{h}(t)\left(u_{0 h}\right)$ and $U_{n}=\mathscr{T}_{h k}^{n}\left(u_{0 h}\right)$. We show below that $\mathscr{T}(t)$ has a global attractor $\mathscr{A}$. This means that $\mathscr{A} \subset \dot{H}^{1}$ is a maximal compact invariant set which attracts every bounded subset of $\dot{H}^{1}$. See Hale [9] for the definitions of these terms. We also show that $\mathscr{T}_{h}(t)$ and $\mathscr{T}_{h k}^{n}$ have global attractors $\mathscr{A}_{h}$ and $\mathscr{A}_{h k}$, respectively, in $\dot{S}_{h} \subset \dot{H}^{1}$.

We may think of $\mathscr{T}_{h}(t)$ and $\mathscr{T}_{h k}^{n}$ as perturbations of $\mathscr{T}(t)$, and the purpose of this section is to use our error bounds for solutions with initial data in $\dot{H}^{1}$ to prove a stability property of the perturbed attractors $\mathscr{A}_{h}$ and $\mathscr{A}_{h k}$.

In fact, applying Theorems 6.5 and 7.2 with $u_{0}=u_{0 h} \in \dot{S}_{h} \subset \dot{H}^{1}$, we immediately obtain

Corollary 8.1. Let $r=2$ or $3, R>0$, and let $J \subset(0, \infty)$ be a compact interval. Then, for small $k$, we have

$$
\begin{gathered}
\delta_{h}(R, J) \equiv \sup _{\substack{v \in \dot{S}_{h} \\
\|v\|_{1} \leq R}} \sup _{t \in J}\left\|\mathscr{T}_{h}(t)(v)-\mathscr{T}(t)(v)\right\|_{1} \leq C(R, J) h^{r-1}, \\
\delta_{h k}(R, J) \equiv \sup _{\substack{v \in \dot{S}_{h} \\
\|v\|_{1} \leq R}} \sup _{t_{n} \in J}\left\|\mathscr{T}_{h k}^{n}(v)-\mathscr{T}\left(t_{n}\right)(v)\right\|_{1} \leq C(R, J)\left(h^{r-1}+k\right) .
\end{gathered}
$$

(Note that the constant blows up as $J$ approaches 0 or $\infty$.) Since $\delta_{h}(R, J)$ $\rightarrow 0, \delta_{h k}(R, J) \rightarrow 0$ as $h, k \rightarrow 0$ for any $R, J$, it follows that

$$
d\left(\mathscr{A}_{h}, \mathscr{A}\right) \rightarrow 0, \quad d\left(\mathscr{A}_{h k}, \mathscr{A}\right) \rightarrow 0 \quad \text { as } h, k \rightarrow 0,
$$


see Temam [17, Theorem I.1.2]. Here, $d(A, B)=\sup _{a \in A} \inf _{b \in B}\|a-b\|_{1}$ is the (unsymmetric) semidistance between the sets $A, B$. Thus (8.1) means that for any $\varepsilon>0$ there is $h$ such that $\mathscr{A}_{h}$ lies in an $\varepsilon$-neighborhood of $\mathscr{A}$, or, in the terminology of [9], that $\mathscr{A}_{h}$ is upper semicontinuous at $h=0$.

The idea of the proof of $(8.1)$ is to compare a discrete trajectory $u_{h}(t)$ on time intervals $[N T,(N+1) T], N=1,2, \ldots$, to exact trajectories $u(t)$ with $u(N T)=u_{h}(N T)$ using Corollary 8.1. The length $T$ of these intervals is determined as the time it takes for $\mathscr{A}$ to attract any initial value into a $\frac{1}{2} \varepsilon$ neighborhood of itself. Everything being uniform on bounded sets of $\dot{H}^{1}$, one may conclude that $u_{h}(t)$ belongs to an $\varepsilon$-neighborhood of $\mathscr{A}$ for $h \leq h_{0}=$ $h_{0}(\varepsilon), t \geq T=T(\varepsilon)$.

The same idea of using a nonsmooth data error bound to obtain a result about the long-time behavior of discrete solutions can be found in Heywood and Rannacher [12]. See also Hale, Lin, and Raugel [10] and Kloeden and Lorenz [14] for related results on the upper semicontinuity of attractors.

We conclude this section by demonstrating the existence of the attractors $\mathscr{A}$, $\mathscr{A}_{h}$, and $\mathscr{A}_{h k}$. This follows easily from a general result about asymptotically smooth gradient systems, see Hale [9, Theorem 3.8.5]. We verify the assumptions of this theorem.

First we note that $\mathscr{T}(t)$ is a $C^{1}$-semigroup in $\dot{H}^{1}$. This means that for fixed $t$ the mapping $u_{0} \mapsto \mathscr{T}(t)\left(u_{0}\right)$ is Fréchet differentiable, which is easily proved using the techniques of the proof of Theorem 4.1 in the supplement. Next we note that the smoothing property of $\mathscr{T}(t)$ obtained in Theorem 4.1 implies that $\mathscr{T}(t)$ is completely continuous. This implies that $\mathscr{T}(t)$ is asymptotically smooth and that all positive orbits $\gamma^{+}(v)=\{\mathscr{T}(t) v: t \geq 0\}$ are precompact (see [9, Corollary 3.2.2, Lemma 3.2.1]). We also note that $\mathscr{T}(t)$ is a gradient system, i.e., it is a $C^{1}$-semigroup with the additional properties:

(1) each bounded positive orbit is precompact;

(2) there is a Ljapunov functional for $\mathscr{T}(t)$, i.e., there is a continuous mapping $V: \dot{H}^{1} \rightarrow \mathbf{R}$ such that

(3) $V$ is bounded below;

(4) $V(u) \rightarrow \infty$ as $|u|_{1} \rightarrow \infty$;

(5) $t \mapsto V(\mathscr{T}(t) v)$ is nondecreasing;

(6) if $v$ is such that $V(\mathscr{T}(t) v)=V(v)$ for all $t$, then $v$ is an equilibrium point of $\mathscr{T}(t)$.

We have already verified (1). Moreover, we saw in $\S 2$ that $V(v)=\frac{1}{2}|v|_{1}^{2}+$ $\int_{\Omega} \psi(v) d x$ is a Ljapunov functional for $\mathscr{T}(t)$.

Finally, we need to check that the set of equilibrium points $\mathscr{E}$ of $\mathscr{T}(t)$ is bounded in $\dot{H}^{1}$. To see this, let $v \in \mathscr{E}$. Then $A v+P \phi(v)=0$, so that $|v|_{1}^{2}+(\phi(v), v)=0$. Using (3.7) and (2.4), we get

$$
|v|_{1}^{2}+c_{0}\|v\|_{L_{2 p}}^{2 p} \leq C+\frac{1}{2} \beta^{2}\|v\|^{2} \leq C\left(1+\|v\|_{L_{2 p}}^{2}\right)
$$

which shows that $|v|_{1} \leq C$.

We are now in a position to apply [9, Theorem 3.8.5]. We conclude that $\mathscr{T}(t)$ has a global attractor $\mathscr{A}$. Moreover, the attractor is connected and equal to the unstable manifold of the set $\mathscr{E}$. Similar arguments apply to $\mathscr{T}_{h}(t)$ and $\mathscr{T}_{h k}^{n}$. In this case the complete continuity is automatic by finite dimensionality. 


\section{BIBLIOGRAPHY}

1. J. W. Cahn and J. E. Hilliard, Free energy of a nonuniform system I. Interfacial free energy, J. Chem. Phys. 28 (1958), 258-267.

2. M. Crouzeix and V. Thomée, On the discretization in time of semilinear parabolic equations with nonsmooth initial data, Math. Comp. 49 (1987), 359-377.

3. M. Crouzeix, V. Thomée, and L. B. Wahlbin, Error estimates for spatially discrete approximations of semilinear parabolic equations with initial data of low regularity, Math. Comp. 53 (1989), 25-41.

4. Q. Du, Finite element solution of a continuum model of phase separation, preprint.

5. Q. Du and R. A. Nicolaides, Numerical analysis of a continuum model for phase transition, Research Report No. 88-23, Department of Mathematics, Carnegie Mellon University, 1988.

6. C. M. Elliott and D. A. French, A nonconforming finite-element method for the two-dimensional Cahn-Hilliard equation, SIAM J. Numer. Anal. 26 (1989), 884-903.

7. C. M. Elliott, D. A. French, and F. A. Milner, A second order splitting method for the Cahn-Hilliard equation, Numer. Math. 54 (1989), 575-590.

8. C. M. Elliott and S.-M. Zheng, On the Cahn-Hilliard equation, Arch. Rational Mech. Anal. 96 (1986), 339-357.

9. J. K. Hale, Asymptotic behavior of dissipative systems, Math. Surveys and Monos., vol. 25, Amer. Math. Soc., Providence, RI, 1988.

10. J. K. Hale, X.-B. Lin, and G. Raugel, Upper semicontinuity of attractors for approximations of semigroups and partial differential equations, Math. Comp. 50 (1988), 89-123.

11. H.-P. Helfrich, Error estimates for semidiscrete Galerkin type approximations for semilinear evolution equations with nonsmooth initial data, Numer. Math. 51 (1987), 559-569.

12. J. G. Heywood and R. Rannacher, Finite element approximation of the nonstationary Navier-Stokes problem II. Stability of solutions and error estimates uniform in time, SIAM J. Numer. Anal. 23 (1986), 750-777.

13. C. Johnson, S. Larsson, V. Thomée, and L. B. Wahlbin, Error estimates for spatially discrete approximations of semilinear parabolic equations with nonsmooth initial data, Math. Comp. 49 (1987), 331-357.

14. P. E. Kloeden and J. Lorenz, Lyapunov stability and attractors under discretization, Differential Equations, Proceedings of the EQUADIFF Conference (C. M. Dafermos, G. Ladas, and G. Papanicolaou, eds.), Marcel Dekker, 1989, pp. 361-368.

15. B. Nicolaenko, B. Scheurer, and R. Temam, Some global dynamical properties of a class of pattern formation equations, Comm. Partial Differential Equations 14 (1989), 245-297.

16. A. Pazy, Semigroups of linear operators and applications to partial differential equations, Springer-Verlag, 1983.

17. R. Temam, Infinite-dimensional dynamical systems in mechanics and physics, SpringerVerlag, 1988.

18. V. Thomée, Galerkin finite element methods for parabolic problems, Lecture Notes in Math., vol. 1054, Springer-Verlag, Berlin and New York, 1984.

19. V. Thomée and L. B. Wahlbin, On Galerkin methods in semilinear parabolic problems, SIAM J. Numer. Anal. 12 (1975), 378-389.

20. W. von Wahl, On the Cahn-Hilliard equation $u^{\prime}+\Delta^{2} u-\Delta f(u)=0$, Delft Progr. Rep. 10 (1985), 291-310.

21. S.-M. Zheng, Asymptotic behavior of solution to the Cahn-Hilliard equation, Appl. Anal. 23 (1986), 165-184.

School of Mathematical and Physical Sciences, University of Sussex, Brighton BN1 9QH, ENGLAND

E-mail address: mmfc8@cluster.sussex.ac.uk

Department of Mathematics, Chalmers University of Technology, S-412 96 Göteborg, SWEDEN

E-mail address: stig@math.chalmers.se 


\title{
Supplement to \\ ERROR ESTIMATES WITH SMOOTH AND NONSMOOTH DATA FOR A FINITE ELEMENT METHOD \\ FOR THE CAHN-HILLIARD EQUATION
}

\author{
CHARLES M. ELLIOTT AND STIG LARSSON
}

Appendix. In this appendix we present the proof of Theorem 4.1. The proof is based on estimating the right-hand side of the variation of constants formula (2.9) for solutions of (2.5), using the analyticity (4.2) of $E(t)$ together with certain bounds for the nonlinearity $\phi(u)$, and the a priori bound (2.8) for the $H^{1}$ norm of $u$. We begin with the required bounds for $\phi(u)$.

Lemma A.1. Assume that $u, v \in \dot{H}^{1}$ with $|u|_{1},|v|_{1} \leq R$. Then there is a constant $C=C(R)$ such that, under the appropriate regularity assumptions for $w$ and $z$, we have

$$
\begin{aligned}
\left|\phi^{\prime}(u) w\right|_{0} & \leq C|w|_{1}, \\
|\phi(u)-\phi(v)|_{0} & \leq C|u-v|_{1}, \\
|\phi(u)|_{0} & \leq C \\
\left|A P\left(\phi^{\prime}(u) w\right)\right|_{0} & \leq C\left(|w|_{3}+|u|_{3}|w|_{1}\right), \\
|A P(\phi(u)-\phi(v))|_{0} & \leq C\left(|u-v|_{3}+\left(|u|_{3}+|v|_{3}\right)|u-v|_{1}\right), \\
|A P \phi(u)|_{0} & \leq C|u|_{3}, \\
\left|\phi^{\prime \prime}(u) w z\right|_{0} \leq C|w|_{1}|z|_{1}, & \leq\left(|w|_{1}|z|_{3}+|w|_{3}|z|_{1}+|u|_{3}|w|_{1}|z|_{1}\right), \\
\left|A P\left(\phi^{\prime \prime}(u) w z\right)\right|_{0} & \leq C\left(|w|_{1}|u-v|_{3}+|w|_{3}|u-v|_{1}\right. \\
& \left.+\left(|u|_{3}+|v|_{3}\right)|w|_{1}|u-v|_{1}\right) .
\end{aligned}
$$

Proof. We only demonstrate (A.8) from which (A.9) readily follows. The bound (A.4) is proved in a similar way, and (A.5), (A.6) then follow. The same is true of (A.1)-(A.3) and (A.7). We present the proof for the case $d=3$; the case $d \leq 2$ is analogous.

For the proof of (A.8) we first note that

$$
\|\Delta(f g)\|_{L_{2}} \leq\|\Delta f\|_{L_{6}}\|g\|_{L_{3}}+2\|\nabla f\|_{L_{6}}\|\nabla g\|_{L_{3}}+\|f\|_{L_{6}}\|\Delta g\|_{L_{3}} .
$$



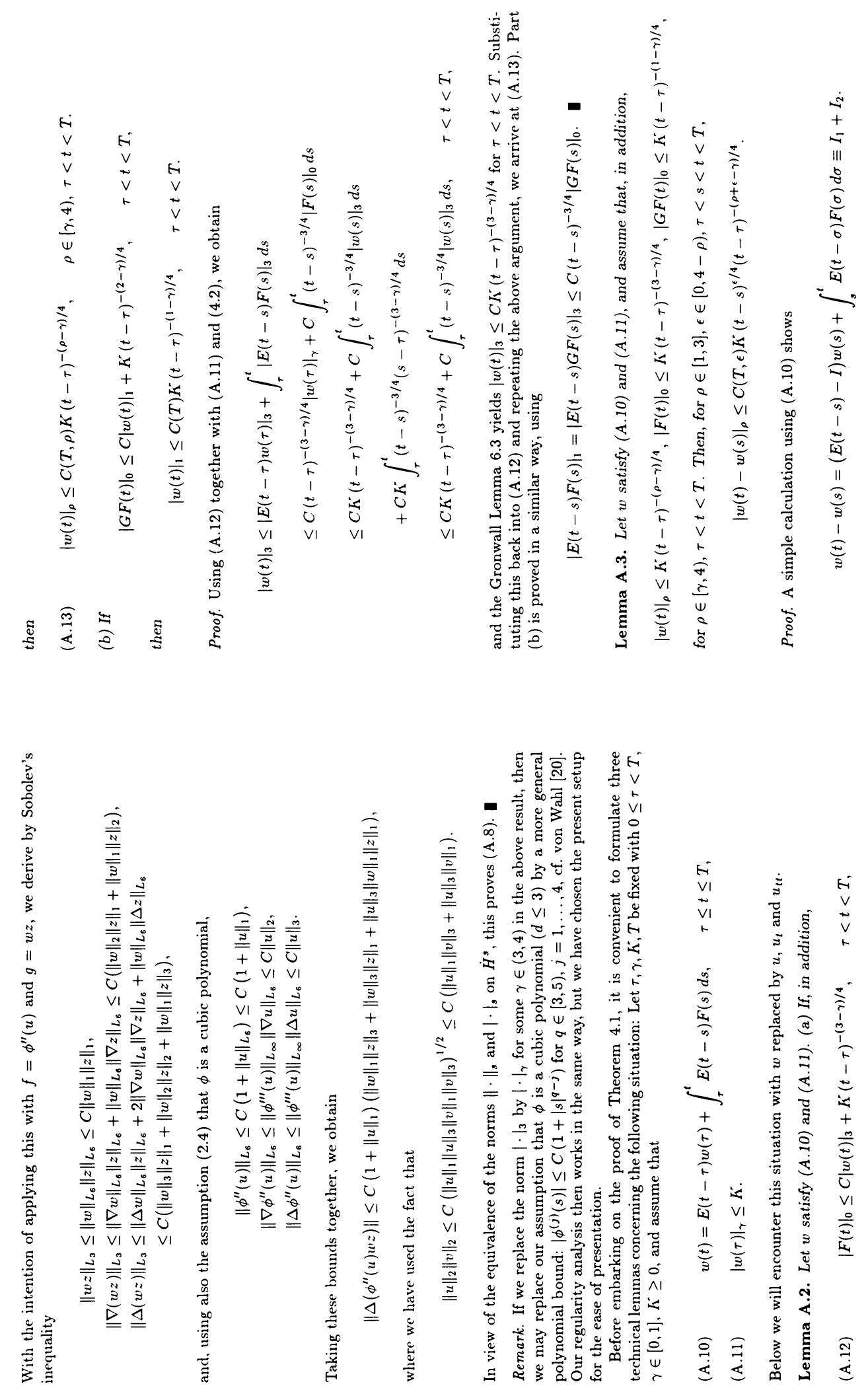


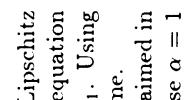
今

- 0

0
0
4

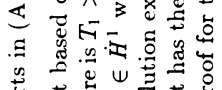

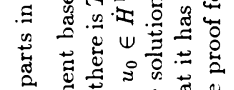

s.

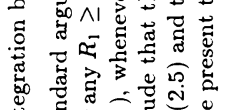

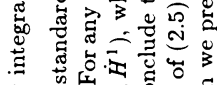

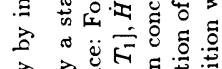

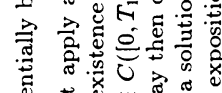

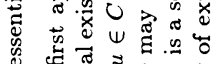

作

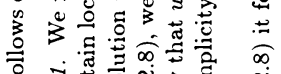

क्ष

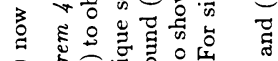

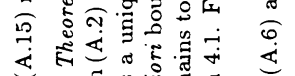

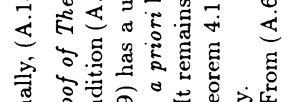

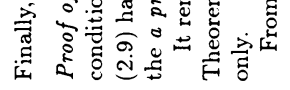

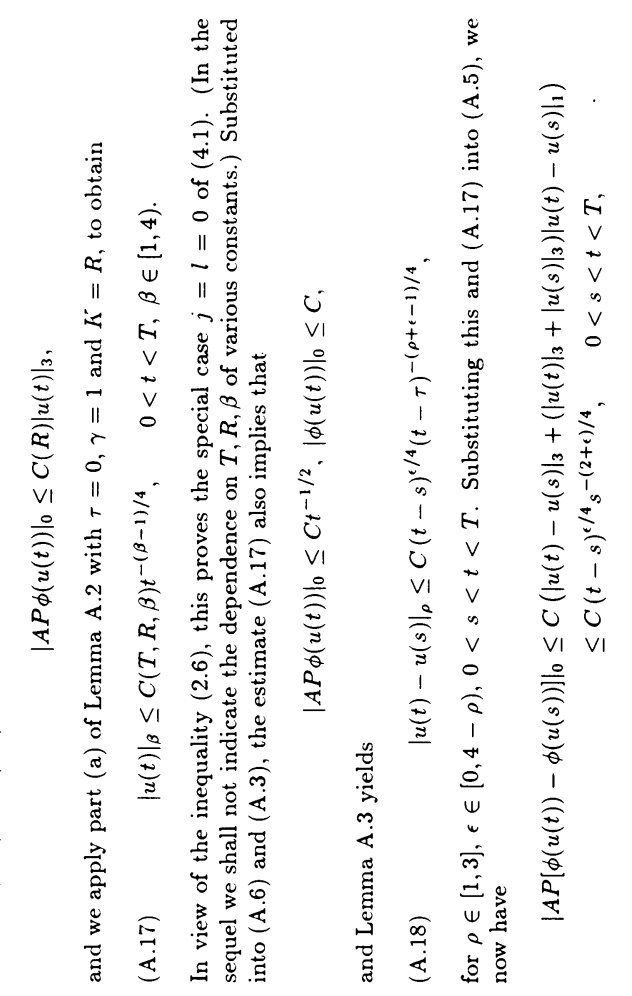

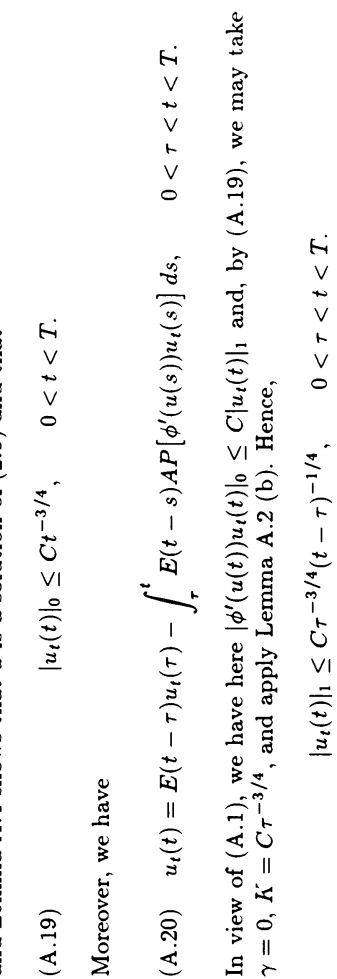

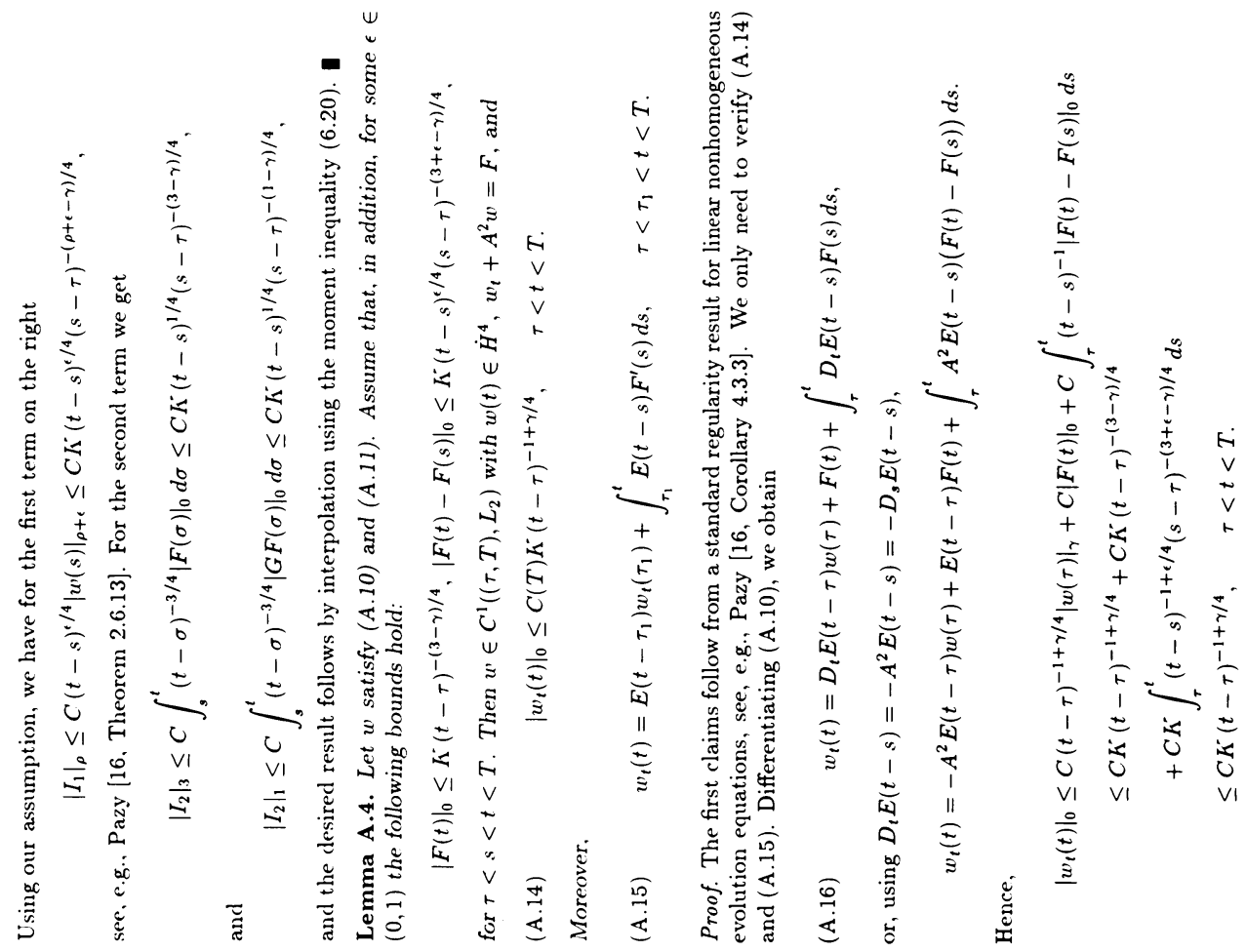



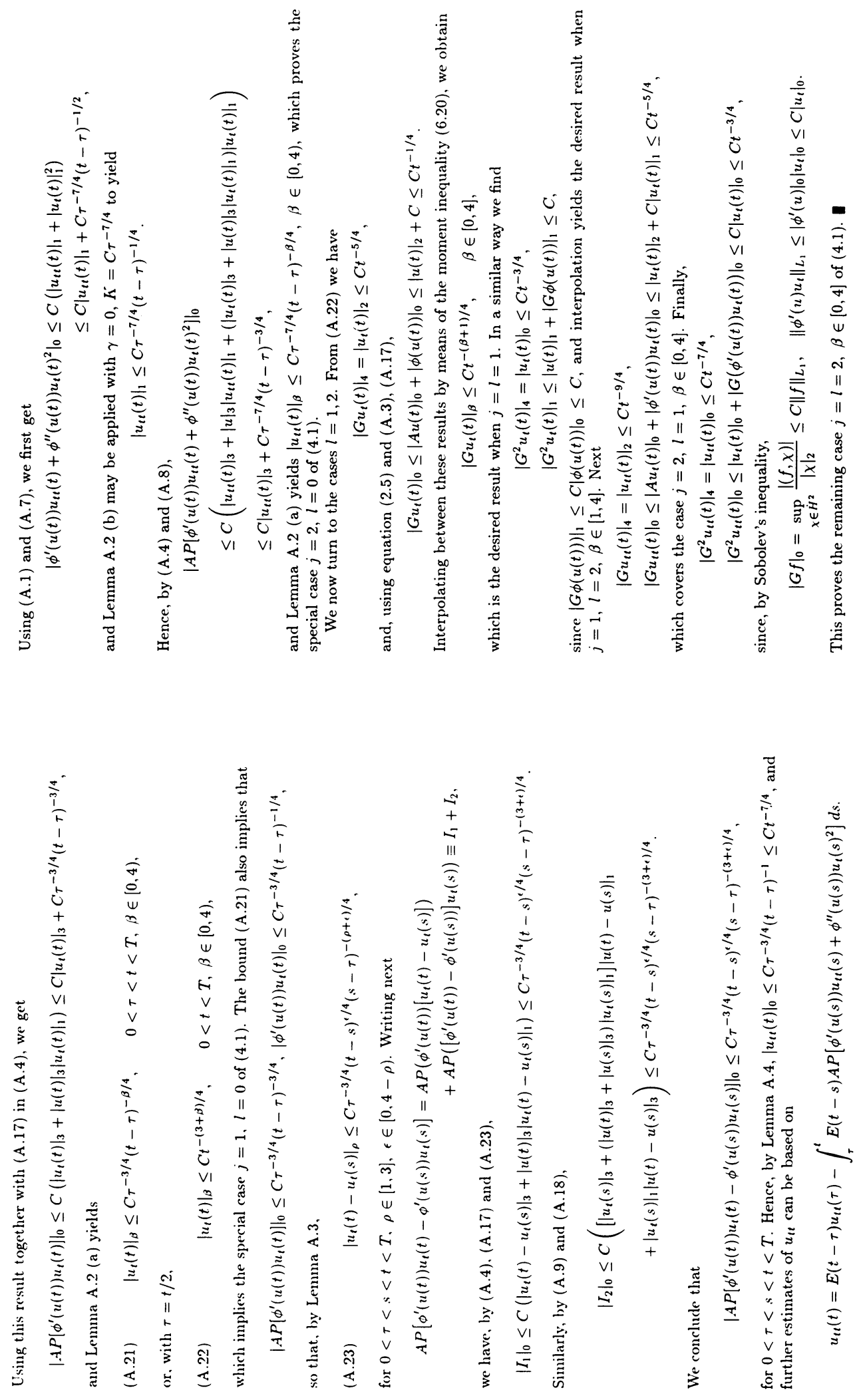